\title{
Arbitrage-free discretization of lognormal forward Libor and swap rate models
}

\author{
Paul Glasserman ${ }^{1}$, Xiaoliang Zhao ${ }^{2}$ \\ 1 Graduate School of Business, Columbia University, Uris Hall, 3022 Broadway, Room 403, \\ New York, NY 10027-6902, USA (e-mail: pg20@columbia.edu) \\ 2 Department of Statistics, Columbia University, New York, NY 10027, USA \\ (e-mail: zhao@stat.columbia.edu)
}

\begin{abstract}
An important recent development in the pricing of interest rate derivatives is the emergence of models that incorporate lognormal volatilities for forward Libor or forward swap rates while keeping interest rates stable. These market models have three attractive features: they preclude arbitrage among bonds, they keep rates positive, and, most distinctively, they price caps or swaptions according to Black's formula, thus allowing automatic calibration to market data. But these features of continuous-time formulations are easily lost when the models are discretized for simulation. We introduce methods for discretizing these models giving particular attention to precluding arbitrage among bonds and to keeping interest rates positive even after discretization. These methods transform the Libor or swap rates to positive martingales, discretize the martingales, and then recover the Libor and swap rates from these discretized variables, rather than discretizing the rates themselves. Choosing the martingales proportional to differences of ratios of bond prices to numeraire prices turns out to be particularly convenient and effective. We can choose the discretization to price one caplet of arbitrary maturity without discretization error. We numerically investigate the accuracy of other caplet and swaption prices as a gauge of how closely a model calibrated to implied volatilities reproduces market prices. Numerical results indicate that several of the methods proposed here often outperform more standard discretizations.
\end{abstract}

Key words: Interest rate models, Monte Carlo simulation, market models

JEL Classification: G14, E43

Mathematics Subject Classification (1991): 60G42, 60G44, 60G15, 60J60, $65 \mathrm{C} 05$

Manuscript received: March 1998; final version received: January 1999 


\section{Introduction}

A major development in the modeling of interest rates for pricing term structure derivatives is the emergence of models that incorporate lognormal volatilities for forward rates while keeping rates stable. It was noted by Heath, Jarrow, and Morton [12] that in the general class of models they developed based on continuously compounded forward rates, lognormal volatilities lead to rates that become infinite in finite time with positive probability. By working instead with various types of discretely compounded rates, Sandmann and Sondermann [23, 24], Brace et al. [5], Goldys et al. [10], Miltersen et al. [19], Musiela and Rutkowski [21], and Jamshidian $[14,15]$ have overcome this difficulty and developed well-posed models that indeed admit deterministic diffusion coefficients for the logarithms of forward rates - i.e., lognormal volatilities. The rates themselves are not simultaneously lognormal, but each becomes lognormal under an appropriate change of measure.

This class of models - often referred to as market models because of their consistency with market conventions - have three principal attractions:

○ they preclude arbitrage among bonds (and just as in the HJM [12] framework this means that the drift is determined once the volatilities are specified);

o they keep rates positive (a consequence of the lognormal form of the volatility that further precludes arbitrage between bonds and cash);

o they price caplets or swaptions according to Black's [3] formula, consistent with market practice.

The first property corresponds to what Musiela and Rutkowski [20] call a weak no-arbitrage condition and the first two together make up their full no-arbitrage condition. The last feature means that the models are easily calibrated to market data. Market participants quote caplet and swaption prices according to their Black implied volatility; if these implied volatilities are used as inputs to a market model, market prices are recovered exactly.

These attractive properties must, however, be understood as features of continuous-time models. (Though discretely compounded, the forward rates evolve continuously). Pricing complex path-dependent instruments in these models typically requires numerical computation and thus discretization. A casual discretization can easily lead to a model without any of the three attractive properties identified above. Since it is ultimately the discretized model that is used for pricing, the theoretical advantages of the continuous-time models are potentially lost in practice. The gap between the discretized and continuous models can be substantial because rather coarse time discretizations (e.g., with an increment of three months) are frequently used in practice.

This paper develops discretizations of lognormal forward Libor and forward swap rates that preserve some, though not all of the attractive features of the continuous-time formulations, and appear to be substantially better than naive discretizations in several respects. We put particular emphasis on discretizations 
ensuring that bond prices deflated by a numeraire asset are martingales, since this is the key condition for the absence of arbitrage.

There is a well-established practice in the derivatives industry of making adjustments to discretized versions of continuous-time models to keep them arbitrage-free. In the open literature, such adjustments are commonly found in binomial models (e.g., [11]) where the discretization is in both time and space. They seem to be less commonly employed in the Monte Carlo setting, where the discretization may be in time alone. In simulating an HJM model using an Euler discretization, a simple adjustment to the drift (reviewed in Sect.2) keeps the discrete model arbitrage-free; this adjustment appears to be widely known in the industry. It may be viewed as a special case of a general strategy of solving for the correct higher-order adjustment to an otherwise straightforward discretization.

For reasons that will be made clear in Sect.2, this strategy is inapplicable to the simulation of forward Libor and forward swap rates; depending on the discretization used, the desired adjustment may not exist or may be intractable. We therefore develop a different strategy. For each model, we find a change of variables that can be discretized in an arbitrage-free way and simulate those variables instead, easily recovering the original Libor and swap rates along the way. For example, the variables may be ratios of bond prices to the price of a numeraire asset, linear combinations of these ratios, or logarithms of linear combinations. In several cases, the new variables are martingales if and only if the model is arbitrage-free. This obviates the need to make a higher-order adjustment to the drift to keep the model arbitrage-free — both the discrete and continuous drift are fixed at zero. We also show how to develop discretizations that keep Libor and swap rates positive. And while it does not seem possible to simultaneously price caplets of all maturities without discretization error, we show how to simulate forward Libor rates to eliminate bias in any one caplet (not necessarily the last). This flexibility may prove useful in adapting the choice of discretization to the instrument to be priced.

There is, of course, a large literature on discretization schemes for stochastic differential equations that accelerate convergence to continuous-time limits; see, e.g., Kloeden and Platen [17]. We view our approach as a complement rather than an alternative to these methods. For example, although we detail only Euler discretizations of our transformed variables, one could also consider higher-order discretizations like those of Milstein [18] and Talay [26]. This would potentially further reduce discretization error while continuing to preclude arbitrage. At the same time, simply applying a higher-order discretization to the original continuous-time equations would not by itself make the discretized process arbitrage-free.

The rest of the paper is organized as follows. Section 2 reviews background on lognormal models of forward Libor and the discretization of continuous-time term structure models. We work within the discrete-tenor formulation of Jamshidian $[14,15]$ and Musiela and Rutkowski $[20,21]$ because it is the best suited for simulation. Section 3 develops discretizations in the terminal measure used by Brace et al. [5] and Musiela and Rutkowski [20, 21], in the spot Libor measure 
introduced by Jamshidian [14, 15], and in a hybrid that provides the flexibility to choose the bias-free caplet. Section 4 carries out a similar analysis for swap rates, first discretizing in the terminal measure, then in the spot Libor measure. In Sect. 5, we numerically compare our discretizations with more standard approaches. We also take the arbitrage-free requirement one step further by enforcing it in finite samples. We argue that the feasibility of such a finite-sample adjustment depends critically on our discretization approach and observe through numerical examples that it can lead to substantial variance reduction. All proofs are deferred to an appendix.

\section{Preliminaries on lognormal Libor models}

We begin with a brief review of discrete-tenor formulations of Libor market models based on finitely many bonds, as developed by Jamshidian [14, 15] and Musiela and Rutkowski $[20,21]$. The tenor structure is a finite set of dates

$$
0=T_{0}<T_{1}<\cdots<T_{N}<T_{N+1}
$$

representing maturities spaced, e.g., three months or six months apart. We will assume throughout that the day-count fractions $\delta_{i} \triangleq T_{i+1}-T_{i}, i=0, \ldots, N$, are all equal to a fixed $\delta$ (e.g., $\delta=0.25$ years). In practice, day-count conventions would make these slightly different; we use a fixed $\delta$ merely to lighten the notation. Define a right-continuous function $\eta:\left[0, T_{N+1}\right) \rightarrow\{1, \ldots, N+1\}$ by taking $\eta(t)$ to be the unique integer satisfying

$$
T_{\eta(t)-1} \leq t<T_{\eta(t)}
$$

Associated with each tenor date $T_{i}$ is a zero-coupon bond maturing at that date; $B_{i}(t)$ is the price of that bond at time $t \in\left[0, T_{i}\right]$ and $B_{i}\left(T_{i}\right)=1$.

The forward Libor rate at time $t$ for the accrual period $\left[T_{i}, T_{i+1}\right], t \leq T_{i}$ is

$$
L_{i}(t)=\frac{1}{\delta}\left(\frac{B_{i}(t)}{B_{i+1}(t)}-1\right), \quad i=1, \ldots, N
$$

It is at times notationally convenient to extend the definition of $L_{i}$ beyond the $i$ th tenor date; we do so by setting $L_{i}(t)=L_{i}\left(T_{i}\right)$ for $t>T_{i}$. At a tenor date $T_{i}$ the price of any bond $B_{n}, n>i$, that has not yet matured is given by

$$
B_{n}\left(T_{i}\right)=\prod_{j=i}^{n-1} \frac{1}{1+\delta L_{j}\left(T_{i}\right)}
$$

more generally, at an arbitrary time $t<T_{n}$ we have

$$
B_{n}(t)=B_{\eta(t)}(t) \prod_{j=\eta(t)}^{n-1} \frac{1}{1+\delta L_{j}(t)} .
$$


As discussed by Jamshidian [14], this reveals an indeterminacy in the model: the bond prices cannot be fully recovered from the forward Libor rates alone, because between tenor dates the forward Libor rates do not specify how to discount back from the next tenor date to the current time. The indeterminacy is removed by the choice of numeraire asset; different choices of numeraire lead to different constructions of the forward Libor processes.

In the constructions we consider, the forward Libor processes are determined by a stochastic differential equation of the form

$$
\frac{d L_{n}(t)}{L_{n}(t)}=\ldots d t+\lambda_{n}(t) d W_{t}, \quad n=1, \ldots, N
$$

in which $W$ is a standard $d$-dimensional Brownian motion, the $L_{n}(0)$ are deterministic, and $\lambda_{n}(t)$ are bounded, deterministic $d$-dimensional row vectors having at most finitely many discontinuities. This is what is meant by a lognormal specification of the volatility. The drift in (3) is determined by the choice of numeraire. (Throughout, we use $W_{t}$ to denote a standard Brownian motion under the measure relevant to the context, rather than introduce a separate symbol for Brownian motion under each measure. This notational simplification should not cause confusion because we seldom consider more than one measure at a time.) If $M$ is a strictly positive semimartingale, then to construct forward Libor in the measure associated with numeraire $M$ is to choose the drift in (3) so that the deflated bond prices

$$
D_{n}(t) \triangleq \frac{B_{n}(t)}{M_{t}}, \quad n=1, \ldots, N+1, \quad \text { are martingales }
$$

which then precludes arbitrage from trades among the bonds. This is the weak noarbitrage condition of Musiela and Rutkowski [20]. See Musiela and Rutkowski [20, 21] and Jamshidian [15] for a thorough development of these issues, and see Duffie [7] and El Karoui et al. [8] for background on changes of numeraire and changes of measure.

Taking $M_{t}=B_{N+1}(t)$ leads to the construction of forward Libor in the terminal (or forward) measure used by Musiela and Rutkowski [20], in which

$$
\frac{d L_{n}(t)}{L_{n}(t)}=-\sum_{i=n+1}^{N} \frac{\delta \lambda_{n}(t) \lambda_{i}(t)^{\prime} L_{i}(t)}{1+\delta L_{i}(t)} d t+\lambda_{n}(t) d W_{t}, \quad n=1, \ldots, N
$$

and in which the deflated bond prices become (cf. (2))

$$
D_{n}(t)=\prod_{j=n}^{N}\left(1+\delta L_{j}(t)\right), \quad n=1, \ldots, N+1 .
$$

Under this measure, the time- 0 price of a security paying $\zeta$ at time $T_{n}$ is

$$
B_{N+1}(0) E\left[\zeta D_{n}\left(T_{n}\right)\right]=B_{N+1}(0) E\left[\zeta \prod_{j=n}^{N}\left(1+\delta L_{j}\left(T_{n}\right)\right)\right]
$$


(Here and throughout, we use $E$ to denote expectation under whatever measure is relevant to the context - in this case, the terminal measure.)

Jamshidian $[14,15]$ introduces the numeraire

$$
B^{*}(t)=\frac{B_{\eta(t)}(t)}{B_{1}(0)} \prod_{j=1}^{\eta(t)-1} \frac{B_{j}\left(T_{j}\right)}{B_{j+1}\left(T_{j}\right)}
$$

which may be interpreted as the result of buying $1 / B_{1}(0)$ bonds at time 0 maturing at $T_{1}$, and then at each tenor date selling the bonds that matured and investing the proceeds in the bond that matures next. (Jamshidian's $\eta$ is left-continuous but for discretization the right-continuous version will be preferable.) This is thus a discretely compounded analog of the money market account that gives rise to the usual risk-neutral measure in, e.g., Heath et al. [12] and numerous other settings. From $B^{*}$, Jamshidian defines the spot Libor measure in which

$$
\frac{d L_{n}(t)}{L_{n}(t)}=\sum_{i=\eta(t)}^{n} \frac{\delta \lambda_{n}(t) \lambda_{i}(t)^{\prime} L_{i}(t)}{1+\delta L_{i}(t)} d t+\lambda_{n}(t) d W_{t}, \quad n=1, \ldots, N
$$

The deflated bond prices become (cf. (2))

$$
\begin{aligned}
D_{n}(t) & =B_{1}(0) \prod_{j=1}^{n-1} \frac{1}{1+\delta L_{j}(t)}, \quad n=1, \ldots, N+1 \\
& =B_{1}(0) \prod_{j=1}^{\eta(t)-1} \frac{1}{1+\delta L_{j}\left(T_{j}\right)} \prod_{j=\eta(t)}^{n-1} \frac{1}{1+\delta L_{j}(t)}
\end{aligned}
$$

and the time- 0 price of a security paying $\zeta$ at time $T_{n}$ is

$$
E\left[\zeta D_{n}\left(T_{n}\right)\right]=B_{1}(0) E\left[\zeta \prod_{j=1}^{n-1} \frac{1}{1+\delta L_{j}\left(T_{j}\right)}\right] .
$$

In Sect. 3.3, we introduce a hybrid of these two numeraires.

In a Monte Carlo implementation, we deal not with the continuous-time processes $L_{n}$ but with some approximation $\hat{L}_{n}$ defined on a finite set of times $0=t_{0}<t_{1}<\cdots<t_{k}$ and then possibly extended to all times in $\left[0, t_{k}\right]$ by interpolation. For simplicity, we will take the $t_{i}$ to be evenly spaced and further assume that their common spacing $h$ divides the tenor spacing $\delta$. This ensures that the tenor dates are among the simulated dates without burdening the notation. We call a discretization arbitrage-free if the discrete deflated bond prices $\hat{D}_{n}$ defined by replacing $L_{n}$ with $\hat{L}_{n}$ in (6) or (9) are discrete-parameter positive martingales on $\{0, h, 2 h, \ldots\}$.

This condition is by no means automatically satisfied. In particular, it is violated by the standard Euler scheme

$$
\hat{L}_{n}((j+1) h)=\hat{L}_{n}(j h)+\hat{L}_{n}(j h) \mu_{n}(j h) h+\hat{L}_{n}(j h) \lambda_{n}(j h)\left[W_{(j+1) h}-W_{j h}\right],
$$


(with $\mu_{n}$ the drift in either (5) or (8)) and also by the discretization

$$
\begin{aligned}
\hat{L}_{n}((j+1) h)= & \hat{L}_{n}(j h) \\
& \times \exp \left(\left[\mu_{n}(j h)-\frac{1}{2} \lambda_{n}(j h) \lambda_{n}(j h)^{\prime}\right] h+\lambda_{n}(j h)\left[W_{(j+1) h}-W_{j h}\right]\right),
\end{aligned}
$$

which corresponds to an Euler scheme for $\log L_{n}$. (Because we take $\eta$ to be rightcontinuous, if $j h=T_{k}$ then in computing $\mu_{n}(j h)$ according to (8) the summation starts at $i=k+1$ rather than $i=k$. This is consistent with the recommendation in Sidenius [25].)

Similar issues arise in discretizing other models, but it is informative to contrast this setting with the closely related HJM framework. A continuous-time HJM model (with scalar $W_{t}$ for simplicity) sets

$$
d f_{T}(t)=\mu_{T}(t) d t+\sigma_{T}(t) d W_{t}
$$

with $f_{T}(t)$ the forward rate for $[T, T+d T]$ as of time $t$ and

$$
\mu_{T}(t)=\sigma_{T}(t) \int_{t}^{T} \sigma_{u}(t) d u
$$

under the risk-neutral measure. This choice of drift ensures that the deflated bond prices

$$
\exp \left(-\int_{t}^{T} f_{u}(t) d u\right) / \exp \left(\int_{0}^{t} f_{u}(u) d u\right)
$$

are martingales (in $t$ ) under the regularity conditions detailed by Heath et al. [12]. Given a discretization $\hat{f}_{i h}(j h)$ in both time and maturity, the corresponding discrete no-arbitrage condition reduces to

$$
e^{-\hat{f}_{i h}(i h) h} E_{i h}\left[e^{-\sum_{j=i+1}^{k} \hat{f}_{j h}((i+1) h) h}\right]=e^{-\sum_{j=i}^{k} \hat{f}_{j h}(i h) h},
$$

with $E_{i h}$ denoting expectation conditional on the history of the process over $\{0, h, \ldots, i h\}$. This condition will not be met by the Euler scheme

$$
\hat{f}_{j h}((i+1) h)=\hat{f}_{j h}(i h)+\tilde{\mu}_{j h}(i h) h+\sigma_{j h}(i h) \sqrt{h} \xi_{i+1},
$$

with $\tilde{\mu}$ defined by replacing integration in (13) by summation and with $\sqrt{h} \xi_{i+1}=$ $\left[W_{(i+1) h}-W_{i h}\right]$. Nevertheless, starting from a specification of the forward rate volatilities and inductively enforcing (14) one can solve for the $\hat{\mu}$ that satisfies (14) when the forward rates are simulated via a modified Euler scheme that replaces $\tilde{\mu}$ with $\hat{\mu}$ in (15). The appropriate modified drift is

$$
\hat{\mu}_{j h}(i h)=\frac{h}{2}\left[\left(\sum_{k=i+1}^{j} \sigma_{k h}(i h)\right)^{2}-\left(\sum_{k=i+1}^{j-1} \sigma_{k h}(i h)\right)^{2}\right] ;
$$

this is a straightforward consequence of the fact that the conditional expectation in (14) involves only the expectation of the exponential of normal random variables, 
which is available explicitly. Formally letting $h \rightarrow 0$ in this expression while holding $t=i h$ and $T=j h$ yields

$$
\frac{1}{2} \frac{d}{d T}\left[\int_{t}^{T} \sigma_{u}(t) d u\right]^{2}=\sigma_{T}(t) \int_{t}^{T} \sigma_{u}(t) d u \equiv \mu_{T}(t) .
$$

The modification to the drift is therefore negligible as $h \rightarrow 0$ but for $h>0$ is just enough to keep the discretized model arbitrage-free. This adjustment is derived in Andersen [1] (in a more general form that does not assume the same discretization for calendar time and maturity) and in Hull [13].

An attempt to apply a similar adjustment to a forward Libor process is quickly defeated. To make the discretization of (9) a martingale by replacing the $\mu_{n}$ in (11) with some $\hat{\mu}_{n}$ we would need in particular (taking $n=2$ )

$$
E\left[\frac{1}{1+\delta L_{1}(0)\left(1+\hat{\mu}_{1}(0) h+\sqrt{h} \lambda_{1}(0) \xi_{1}\right)}\right]=\frac{1}{1+\delta L_{1}(0)}, \quad \xi_{1} \sim N(0, I)
$$

but the expectation on the left is infinite for all choices of $\hat{\mu}_{1}(0)$. Using (12) (again with $\mu_{n}$ replaced by some $\hat{\mu}_{n}$ ) entails, at a minimum, quantities of the form $E[1 /(1+\exp (X))]$ with $X \sim N(a, b)$ - an expression involving three infinite trigonometric series (equation (56) of Johnson [16]). The necessary adjustment quickly becomes intractable. One could replace the normal $\xi_{i}$ with, say, Bernoulli increments without affecting the validity of the Euler scheme ([17, p.458]), but even this does not lead to a tractable drift correction. Enforcing conditions on the apparently simpler case of (6) becomes unmanageable too. A different approach is required.

\section{Discretization of forward Libor}

\subsection{Terminal measure}

Rather than discretize forward Libor and try to enforce the martingale property indirectly, we simulate suitable martingales directly and then recover discretized Libor from these. There is some flexibility in the choice of martingales and this can be used to advantage; differences of deflated bond prices will turn out to be particularly effective and convenient. We begin with the specification of forward Libor in (5), corresponding to the dynamics under the terminal measure. Set

$$
X_{n}(t)=L_{n}(t) \prod_{i=n+1}^{N}\left(1+\delta L_{i}(t)\right)=\frac{1}{\delta}\left(D_{n}(t)-D_{n+1}(t)\right), \quad n=1, \ldots, N .
$$

Lemma 1 Each $X_{n}$ is a martingale and satisfies

$$
\frac{d X_{n}}{X_{n}}=\left(\lambda_{n}+\sum_{j=n+1}^{N} \frac{\delta X_{j} \lambda_{j}}{1+\delta X_{j}+\cdots+\delta X_{N}}\right) d W
$$


Also,

$$
L_{n}=\frac{X_{n}}{1+\delta X_{n+1}+\cdots+\delta X_{N}} .
$$

This immediately suggests an algorithm for arbitrage-free simulation: simulate a discretization of the $\hat{X}_{n}$, ensuring that the discretized process remains a martingale, and then use (20) to define

$$
\hat{L}_{n}=\frac{\hat{X}_{n}}{1+\delta \hat{X}_{n+1}+\cdots+\delta \hat{X}_{N}} .
$$

The discretized deflated bond prices (cf. (6))

$$
\hat{D}_{n}=\prod_{j=n}^{N}\left(1+\delta \hat{L}_{j}\right) \equiv 1+\delta \sum_{j=n}^{N} \hat{X}_{j}
$$

are automatically positive martingales because the $\hat{X}_{j}$ are. Enforcing the martingale property on $\hat{X}_{n}$ is straightforward and circumvents the need to make a drift adjustment to the $\hat{L}_{n}$. Indeed, even an Euler discretization of $X_{n}$ preserves the martingale property. We will see in Theorem 1 below that discretizing $\log X_{n}$ instead will keep the Libor rates positive. We use a modified Euler scheme that accounts for time-varying volatilities. First, let $\Lambda(t)$ be the $N \times d$ matrix with $n$th row equal to $\lambda_{n}(t), n=1, \ldots, N$. With $h$ fixed, let $\hat{\Lambda}(i h)$ denote any solution to the equation

$$
\hat{\Lambda}(i h) \hat{\Lambda}(i h)^{\prime}=\frac{1}{h} \int_{0}^{h} \Lambda(i h+u) \Lambda(i h+u)^{\prime} d u
$$

and let $\hat{\lambda}_{n}(i h)$ denote the $n$th row of $\hat{\Lambda}(i h)$. Now set

$$
\hat{X}_{n}((i+1) h)=\hat{X}_{n}(i h) \exp \left(-\frac{1}{2} \sigma_{\hat{X}_{n}}(i h) \sigma_{\hat{X}_{n}}^{\prime}(i h) h+\sqrt{h} \sigma_{\hat{X}_{n}}(i h) \xi_{i+1}\right)
$$

with

$$
\sigma_{\hat{X}_{n}}=\hat{\lambda}_{n}+\sum_{j=n+1}^{N} \frac{\delta \hat{X}_{j} \hat{\lambda}_{j}}{1+\delta \hat{X}_{j}+\cdots+\delta \hat{X}_{N}},
$$

and $\xi_{1}, \xi_{2}, \ldots$, independent standard normal $d$-dimensional vectors. (The $\xi_{i}$ could be replaced with other random vectors satisfying moment conditions discussed in Kloeden and Platen [17, p.458] and Talay [26, p.307] but normal inputs give a better approximation to the desired distribution, particularly when $h$ is not very small.) This differs slightly from a standard Euler scheme for $\log X_{n}$, which would use $\lambda_{j}(i h), j=n, \ldots, N$, in (25) rather than $\hat{\lambda}_{j}(i h)$. The two would coincide (or could be made to by choosing $\hat{\Lambda}=\Lambda$ ) if the $\lambda_{n}$ were constant functions of time $t$. Over each interval $[i h,(i+1) h)$, the scheme in (25) freezes the stochastic elements of the diffusion coefficient at their values at $i h$ but uses the average over the interval (in the sense of (23)) of the deterministically time-varying elements. In practice, the distinction is unlikely to matter because in calibrating to a finite 
number of cap prices one would typically choose piecewise constant $\lambda_{n}$, for which the two methods are the same. The formulation in (25) is slightly more convenient for our analysis.

In Sect. 1 we noted that the defining property of market models of forward Libor is that they price caplets according Black's formula, the industry convention (cf. [5, 14, 15, 19, 21]). This means that using implied volatilities for the $\lambda_{n}$ automatically calibrates the model to market prices. This, however, is strictly true only for the continuous-time forward Libor process; we examine the extent to which it remains true after discretization.

Let

$$
\begin{aligned}
C(\sigma, K, r, b, T)= & \delta b\left[r \Phi\left(\frac{\log (r / K)+\frac{1}{2} \sigma^{2} T}{\sigma \sqrt{T}}\right)\right. \\
& \left.-K \Phi\left(\frac{\log (r / K)-\frac{1}{2} \sigma^{2} T}{\sigma \sqrt{T}}\right)\right],
\end{aligned}
$$

with $\Phi$ the standard normal cumulative distribution. This is Black's formula for the price at time 0 of a caplet covering the interval $[T, T+\delta]$, settled at time $T+\delta$ and struck at $K$, when the forward rate at time 0 for $[T, T+\delta]$ is $r$, the implied volatility is $\sigma$, and the price of a discount bond maturing at $T+\delta$ is $b$; see, e.g., $[21, \S 15.3]$ or $[22, \S 1.4]$. The expression in square brackets on the right side of (26) evaluates

$$
E\left[\left(r e^{-\frac{1}{2} \sigma^{2} T+\sigma \sqrt{T} \xi}-K\right)^{+}\right], \quad \xi \sim N(0,1) .
$$

In terms of forward Libor, the payoff on a caplet over $\left[T_{n}, T_{n+1}\right]$ (received at time $\left.T_{n+1}\right)$ is $\delta\left(L_{n}\left(T_{n}\right)-K\right)^{+}$. Under the terminal measure $P^{N+1}$ associated with $T_{N+1}$, the time-0 value of a payoff of $\zeta$ (say) at time $T_{n+1}$ is $B_{N+1}(0) E\left[\zeta / B_{N+1}\left(T_{n+1}\right)\right]$. Using superscripts on expectations to emphasize the underlying measure, we have

$$
\begin{aligned}
B_{N+1}(0) E^{N+1}\left[\frac{\zeta}{B_{N+1}\left(T_{n+1}\right)}\right] & =B_{n+1}(0) E^{N+1}\left[\zeta \frac{B_{n+1}\left(T_{n+1}\right) B_{N+1}(0)}{B_{N+1}\left(T_{n+1}\right) B_{n+1}(0)}\right] \\
& \triangleq B_{n+1}(0) E^{n+1}[\zeta]
\end{aligned}
$$

where the new measure $P^{n+1}$ is defined by

$$
\left(\frac{d P^{n+1}}{d P^{N+1}}\right)_{t}=\frac{B_{n+1}(t) B_{N+1}(0)}{B_{N+1}(t) B_{n+1}(0)}=\frac{B_{N+1}(0)}{B_{n+1}(0)} \prod_{j=n+1}^{N}\left(1+\delta L_{j}(t)\right) .
$$

It follows from (28) that $L_{n}$ is a martingale under $P^{n+1}$ because $X_{n}$ is a martingale under $P^{N+1}$ (in fact, $P^{n+1}$ is just the terminal measure associated with $T_{n+1}$ ), so Girsanov's theorem implies that $d L_{n}=\lambda_{n} L_{n} d W$ under $P^{n+1}$ ( $W$ here denoting a standard Brownian motion under $P^{n+1}$ ). Consequently, the price of the $n$th caplet is 


$$
\begin{aligned}
B_{N+1}(0) E^{N+1}\left[\delta\left(L_{n}\left(T_{n}\right)-K\right)^{+} / B_{N+1}\left(T_{n+1}\right)\right] & =\delta B_{n+1}(0) E^{n+1}\left[\left(L_{n}\left(T_{n}\right)-K\right)^{+}\right] \\
& =C\left(\bar{\lambda}_{n}\left(T_{n}\right), K, L_{n}(0), B_{n+1}(0), T_{n}\right),
\end{aligned}
$$

with

$$
\bar{\lambda}_{n}^{2}(t)=\frac{1}{t} \int_{0}^{t} \lambda_{n}(u) \lambda_{n}^{\prime}(u) d u
$$

using (26) and (27).

The pricing rule in the discretized model replaces $D_{n}$ with $\hat{D}_{n}$ in (7). The price of the caplet is

$$
B_{N+1}(0) E\left[\delta\left(\hat{L}\left(T_{n}\right)-K\right)^{+} \prod_{j=n+1}^{N}\left(1+\delta \hat{L}_{j}\left(T_{n+1}\right)\right)\right] .
$$

Under our discretization (but not (11) or (12)), the product inside this expectation (which is simply $\hat{D}_{n+1}$ ) remains a positive martingale even after discretization. Thus, it may still be used to define a new measure, and $\hat{L}_{n}$ is a martingale under this measure (Theorem 1(iii), below). Again, we stress that this is a specific consequence of the scheme in (21) and (24) and would not hold under standard discretizations of forward Libor.

At this point, however, we encounter a fundamental difference between discrete and continuous time. In continuous time, any absolutely continuous change of measure that makes $L_{n}$ a martingale must in fact make $L_{n}$ lognormal. But in discrete time an absolutely continuous change of measure for $\xi_{1}, \xi_{2}, \ldots, \xi_{m}$ may not simply correspond to a change of mean. So, we cannot conclude from the fact that $\hat{L}_{n}$ is a martingale under the new measure that it is lognormal under the new measure. Indeed, to accomplish this would require that both $\log \hat{L}_{n}$ and $\log \hat{D}_{n}$ be linear in the $\xi_{i}$, and this is clearly not possible. Thus, we cannot expect all caplets to be priced simultaneously by Black's formula in a discretized model; but the arbitrage-free discretization above arguably brings us closer to this ideal by at least ensuring that each $\hat{L}_{n}$ is a martingale in the discrete terminal measure associated with $T_{n+1}$. We return to this issue in Sect. 3.3, in part (iv) of Theorem 1, and in the numerical results of Sect. 5.

It remains to address the question of whether the $\hat{L}_{n}$ defined above, in addition to having various desirable properties already noted, actually bear some relation to their ostensive continuous-time counterparts. To formulate a convergence result, it is useful to extend the definition of the discretized variables to the interval $\left[0, T_{N+1}\right]$. Proceeding by induction on $i$, over each interval $[i h,(i+1) h)$ we may construct the solution to

$$
\frac{d \hat{X}_{n}(t)}{\hat{X}_{n}(t)}=\left(\lambda_{n}(t)+\sum_{j=n+1}^{N} \frac{\delta \hat{X}_{j}(i h) \lambda_{j}(t)}{1+\delta \hat{X}_{j}(i h)+\cdots+\delta \hat{X}_{N}(i h)}\right) d W_{t}
$$

and if we take $\sqrt{h} \xi_{i+1}=W_{(i+1) h}-W_{i h}$ then (24) will indeed coincide with the solution to (29) at every grid point $i h$. We can apply (20) to obtain interpolated $\hat{L}_{n}$ from $\hat{X}_{n}$. This makes $\hat{L}$ a random element of $C_{\mathbf{R}^{N}}\left[0, T_{N+1}\right]$, the space of 
continuous functions from $\left[0, T_{N+1}\right]$ to $\mathbf{R}^{N}$. Endow this space with the topology of uniform convergence. Let $\mathscr{P}$ be the measure on this space induced by $\hat{L}$ and $\mathscr{P}$ that induced by $L$. Let $\Rightarrow$ denote weak convergence. We summarize properties of $\hat{L}$ in the following result.

Theorem 1 (i) The discretization (18)-(21) makes the deflated bond prices $\hat{D}$ discrete-time positive martingales and is thus arbitrage-free. (ii) The $\hat{L}_{n}$ remain strictly positive, almost surely. (iii) For each $n,\left\{\hat{L}_{n}(i h), i=1,2, \ldots\right\}$ is a martingale with respect to the measure $\hat{P}_{n+1}$ defined by $d \hat{P}^{n+1}=\left[\left(B_{N+1}(0) \hat{D}_{n+1} / B_{n+1}(0)\right)\right]$ $d P^{N+1}$. (iv) The last caplet is priced without discretization error; i.e.,

$$
B_{N+1}(0) \delta E\left[\left(\hat{L}_{N}\left(T_{N}\right)-K\right)^{+}\right]=C\left(\bar{\lambda}_{N}\left(T_{N}\right), K, L_{N}(0), B_{N+1}(0), T_{N}\right) ;
$$

(v) $\mathscr{P} h \Rightarrow \mathscr{P}$ as $h \rightarrow 0$.

We conclude our discussion of discretization in the terminal measure with some remarks about an alternative discretization. We could enforce the absence of arbitrage by directly discretizing the deflated bond prices $D_{n}$, rather than the $X_{n}$. Observe that

$$
\frac{d D_{n}}{D_{n}}=\sum_{j=n}^{N} \frac{\delta L_{j}}{1+\delta L_{j}} \lambda_{j} d W .
$$

Suppose, for simplicity, that $W_{t}$ and the $\lambda_{j}(t)$ are one-dimensional. This suggests the martingale discretization

$$
\begin{aligned}
\tilde{D}_{n}((i+1) h)= & \tilde{D}_{n}(i h) \exp \left(-\frac{1}{2}\left(\sum_{j=n}^{N} \frac{\delta \tilde{L}_{j}(i h)}{1+\delta \tilde{L}_{j}(i h)} \lambda_{j}(i h)\right)^{2} h\right. \\
& \left.+\sum_{j=n}^{N} \frac{\delta \tilde{L}_{j}(i h)}{1+\delta \tilde{L}_{j}(i h)} \lambda_{j}(i h) h \xi_{i+1}\right)
\end{aligned}
$$

with the $\xi_{i}$ independent standard normals. Using (6), we can solve for the $\tilde{L}_{n}$ to get

$$
\begin{aligned}
& \left(1+\delta \tilde{L}_{n}((i+1) h)\right)= \\
& \quad\left(1+\delta \tilde{L}_{n}(i h)\right) \exp \left(\frac{1}{2}\left(\sum_{j=n+1}^{N} \frac{\delta \tilde{L}_{j}(i h)}{1+\delta \tilde{L}_{j}(i h)} \lambda_{j}(i h)\right)^{2} h\right. \\
& \left.\quad-\frac{1}{2}\left(\sum_{j=n}^{N} \frac{\delta \tilde{L}_{j}(i h)}{1+\delta \tilde{L}_{j}(i h)} \lambda_{j}(i h)\right)^{2} h\right) \\
& \quad \times \exp \left(\frac{\delta \tilde{L}_{n}(i h)}{1+\delta \tilde{L}_{n}(i h)} \lambda_{n}(i h) h \xi_{i+1}\right) .
\end{aligned}
$$


Simulating not $L_{n}$ itself but rather $1+\delta L_{n}$ in this way thus leads to an alternative arbitrage-free discretization. The expression in the exponent

$$
\frac{h}{2}\left[\left(\sum_{j=n+1}^{N} \frac{\delta \tilde{L}_{j}(i h)}{1+\delta \tilde{L}_{j}(i h)} \lambda_{j}(i h)\right)^{2}-\left(\sum_{j=n}^{N} \frac{\delta \tilde{L}_{j}(i h)}{1+\delta \tilde{L}_{j}(i h)} \lambda_{j}(i h)\right)^{2}\right]
$$

is reminiscent of the adjusted discrete HJM drift (16). Indeed, one could view this as the appropriate drift adjustment to keep an Euler scheme for $\log \left(1+\delta L_{n}\right)$ arbitrage-free. A drawback of this discretization, compared to the one based on $X_{n}$, is that while it ensures that $1+\delta L_{n}$ stays positive, it does not ensure that $L_{n}$ itself stays positive. We compare them numerically in Sect. 5.

\subsection{Spot Libor measure}

For discretization in the spot Libor measure, we introduce the variables

$$
\begin{aligned}
Y_{n}(t) & =\frac{1}{1+\delta L_{n}(t)}, \quad V_{n}(t)=\left(1-Y_{n}(t)\right) \prod_{i=1}^{n-1} Y_{i}(t), \quad n=1, \ldots, N, \quad \text { and } \\
V_{N+1} & =\prod_{i=1}^{N} Y_{i}(t)
\end{aligned}
$$

An expression for the deflated bond prices $D_{n}$ was given in (9), from which it follows that $V_{n}(t)=\left(D_{n}(t)-D_{n+1}(t)\right) / B_{1}(0)$. Under our convention that $L_{n}(t)=$ $L_{n}\left(T_{n}\right)$ for $t \geq T_{n}$, the definitions of $Y_{n}, V_{n}$ and $D_{n}$ extends to all of [0, $\left.T_{N+1}\right]$. We record some additional useful formulas in the following lemma.

Lemma 2 Under the spot Libor measure, each $V_{n}, n=1, \ldots, N+1$, is a martingale and satisfies

$\frac{d V_{n}}{V_{n}}=\left[\left(\frac{V_{n}+V_{n-1}+\cdots+V_{1}-1}{V_{n-1}+\cdots+V_{1}-1}\right) \lambda_{n}+\sum_{i=\eta}^{n-1}\left(\frac{V_{i}}{V_{i-1}+\cdots+V_{1}-1}\right) \lambda_{i}\right] d W$

with the convention $\lambda_{N+1} \equiv 0$. Also,

$$
\begin{gathered}
\frac{d D_{n+1}}{D_{n+1}}=\sum_{i=\eta}^{n}\left(\frac{D_{i+1}}{D_{i}}-1\right) \lambda_{i} d W \\
D_{n+1}=B_{1}(0)\left(1-\sum_{i=1}^{n} V_{i}\right)=B_{1}(0)\left(\sum_{i=n+1}^{N+1} V_{i}\right) .
\end{gathered}
$$

and

$$
\begin{aligned}
\delta L_{n}=\frac{D_{n}-D_{n+1}}{D_{n+1}} & =\frac{V_{n}}{1-V_{n}-\cdots-V_{1}} \\
& =\frac{V_{n}}{V_{n+1}+\cdots+V_{N+1}} .
\end{aligned}
$$


At first sight, (31) and (32) may seem problematic; in each case, multiplying both sides by the denominator on the left makes plain that the diffusion coefficient as written fails to be Lipschitz. This however is only a matter of appearance and is easily circumvented. An obvious consequence of (8) and (9) is that $D_{i}>D_{i+1}>$ 0 throughout $\left[0, T_{i}\right]$, so (32) is unchanged if we write it as $d D_{n+1}=D_{n+1} \sigma_{D_{n+1}} d W$, with

$$
\sigma_{D_{n+1}}=-\sum_{i=\eta}^{n} \phi\left(1-\frac{D_{i+1}}{D_{i}}\right) \lambda_{i}
$$

and $\phi(x)=\min \left\{1, x^{+}\right\}$. Similarly, we can use (33) in (31) to write (with the convention $D_{N+2} \equiv 0$ )

$$
\frac{d V_{n}}{V_{n}}=\left[\frac{D_{n+1}}{D_{n}} \lambda_{n}-\sum_{i=\eta}^{n-1} \frac{D_{i}-D_{i+1}}{D_{i}} \lambda_{i}\right] d W,
$$

and thus $d V_{n}=V_{n} \sigma_{V_{n}} d W$ with

$$
\begin{aligned}
\sigma_{V_{n}} & =\left[\phi\left(\frac{D_{n+1}}{D_{n}}\right) \lambda_{n}-\sum_{i=\eta}^{n-1} \phi\left(\frac{D_{i}-D_{i+1}}{D_{i}}\right) \lambda_{i}\right] \\
& =\phi\left(\frac{V_{n}+V_{n-1}+\cdots+V_{1}-1}{V_{n-1}+\cdots+V_{1}-1}\right) \lambda_{n}-\sum_{i=\eta}^{n-1} \phi\left(\frac{V_{i}}{1-V_{i-1}-\cdots-V_{1}}\right) \lambda_{i} .
\end{aligned}
$$

Rewriting the equations this way makes the diffusion coefficients $\sigma_{D_{n}} D_{n}$ and $\sigma_{V_{n}} V_{n}$ Lipschitz functions of the $D_{i}$ and $V_{i}$ respectively without affecting the continuous-time dynamics.

Lemma 2 suggests several possible discretizations. We can set

$$
\hat{V}_{n}((i+1) h)=\hat{V}_{n}(i h) \exp \left(-\frac{1}{2} \sigma_{\hat{V}_{n}}(i h) \sigma_{\hat{V}_{n}}^{\prime}(i h) h+\sigma_{\hat{V}_{n}}(i h) \sqrt{h} \xi_{i+1}\right),
$$

with $\sigma_{\hat{V}_{n}}$ as in (37) but with the $V_{i}$ replaced by $\hat{V}_{i}$ and $\lambda_{i}$ by $\hat{\lambda}_{i}$; or we can set

$$
\tilde{D}_{n}((i+1) h)=\tilde{D}_{n}(i h) \exp \left(-\frac{1}{2} \sigma_{\tilde{D}_{n}}(i h) \sigma_{\tilde{D}_{n}}^{\prime}(i h) h+\sigma_{\tilde{D}_{n}}(i h) \sqrt{h} \xi_{i+1}\right),
$$

with $\sigma_{\tilde{D}_{1}}$ the corresponding quantity from (36). Using (39), we then recover discretized forward Libor rates $\tilde{L}_{n}$ from the first equality in (34). Using (38) and the second equality in (34) we can set

$$
\delta \hat{L}_{n}=\frac{\hat{V}_{n}}{1-\hat{V}_{n}-\cdots-\hat{V}_{1}}, \quad n=1, \ldots, N,
$$

leading (via (9)) to

$$
\hat{D}_{n}=B_{1}(0)\left(\prod_{i=1}^{n-1} \frac{1}{1+\delta \hat{L}_{i}}\right)=B_{1}(0)\left(1-\sum_{i=1}^{n-1} \hat{V}_{i}\right) .
$$


Alternatively, we can use (35) to define

$$
\delta L_{n}^{\prime}=\frac{\hat{V}_{n}}{\hat{V}_{n+1}+\cdots+\hat{V}_{N+1}}, \quad n=1, \ldots, N .
$$

In continuous time, $V_{1}+\cdots+V_{N+1} \equiv 1$ (this is implicit in (34)-(35)), so

$$
D_{n}(t)=B_{1}(0)\left(\prod_{i=1}^{n-1} \frac{1}{1+\delta L_{i}(t)}\right)\left(V_{1}(t)+\cdots+V_{N+1}(t)\right)
$$

is equivalent to the representation of the deflated bond prices in (9). We may therefore choose to define the discrete deflated bond prices as

$$
D_{n}^{\prime}=B_{1}(0)\left(\prod_{i=1}^{n-1} \frac{1}{1+\delta L_{i}^{\prime}}\right)\left(\hat{V}_{1}+\cdots+\hat{V}_{N+1}\right)
$$

which simplifies to

$$
D_{n}^{\prime}=B_{1}(0) \sum_{i=n}^{N+1} \hat{V}_{i}
$$

Depending on whether we use (40) or (42), we replace $D_{n}$ with $\hat{D}_{n}$ or $D_{n}^{\prime}$ in the pricing rule (10).

We summarize properties of the discretizations in this section in the following result. Interpolate $\hat{V}$ and $\tilde{D}$ to continuous time the way we interpolated $\hat{X}$ in the previous section. Let $\hat{\mathscr{P}}, \tilde{\mathscr{R}}$, and $\mathscr{P}_{h}^{\prime}$ be the measures on $C_{\mathbf{R}^{d}}\left[0, T_{N+1}\right]$ associated with the interpolations of $\tilde{L}, \hat{L}$, and $L^{\prime}$ resulting from (34) (40) and (42), respectively, and let $\mathscr{P}$ denote the corresponding measure induced by $L$ under the spot Libor measure.

Theorem 2 (i) The discretizations above make the deflated bond prices $\tilde{D}$ and $\hat{D}$, and $D^{\prime}$ discrete-time martingales. (ii) The $\hat{D}_{n}$ are decreasing in $n$, the $\tilde{D}_{n}$ remain positive, and the $D_{n}^{\prime}$ are both positive and decreasing in $n$. (iii) The $L_{n}^{\prime}$ are positive. (iv) Each $\tilde{L}_{n}$ is a discrete-time martingale with respect to the measure $\tilde{P}_{n+1}$ defined by $d \tilde{P}_{n+1}=\left(B_{1}(0) \tilde{D}_{n+1} / B_{n+1}(0)\right) d P^{*}$, with $P^{*}$ the spot Libor measure. Each $L_{n}^{\prime}$ is a discrete-time martingale with respect to the measure $P_{n+1}^{\prime}$ defined by $d P_{n+1}^{\prime}=\left(B_{1}(0) D_{n+1}^{\prime} / B_{n+1}(0)\right) d P^{*}$. (v) As $h \rightarrow 0$, $\tilde{\mathscr{P}} \Rightarrow \mathscr{P}, \hat{\mathscr{P}} \Rightarrow \mathscr{P}$, and $\mathscr{P}_{h}^{\prime} \Rightarrow \mathscr{P}$.

This result suggests that the discretization of $V_{n}$ combined with (42) and (44) is the most attractive from a theoretical viewpoint. It makes the deflated bond prices positive martingales and it keeps all forward Libor rates positive. Numerical results in Sect. 5, however, indicate that there are sometimes advantages to using $V_{n}$ with (40) and (41). None of the caplets is priced exactly by Black's formula under any of these discretizations, essentially because no $L_{n}$ is a martingale under the spot Libor measure. This motivates the approach of the next section. 


\subsection{A hybrid numeraire}

We saw in Sect. 3.1 that it is possible to discretize forward Libor in a way that prices caplets over $\left[T_{N}, T_{N}+\delta\right]$ without discretization error. By letting the strike vary, we see that this is equivalent to the statement that the distribution of $\hat{L}_{N}\left(T_{N}\right)$ coincides exactly with that of $L_{N}\left(T_{N}\right)$ under the terminal measure (indeed, the distributions match at all grid points). It is possible, however, that one may want to price a Libor derivative that depends on the entire tenor structure $T_{1}, \ldots, T_{N}$ and yet for which one is most concerned about getting the distribution of some intermediate $L_{n}$ correct, rather than $L_{N}$. To accomplish this, we introduce a family of numeraires and an associated family of measures.

For any $m=1, \ldots, N+1$, define

$$
B_{m}^{*}(t)= \begin{cases}B_{m}(t), & t<T_{m} \\ B_{\eta(t)}(t) \prod_{i=m+1}^{\eta(t)} \frac{1}{B_{i}\left(T_{i-1}\right)}, & t \geq T_{m} .\end{cases}
$$

This is the value of a trading strategy that holds one unit of the bond maturing at $T_{m}$ until maturity and at each subsequent tenor date rolls the proceeds over into the next bond to mature. With $m=N+1$ we get the terminal bond and with $m=1$ we get Jamshidian's numeraire (up to a factor of $1 / B_{1}(0)$ ). Thus, (45) may be viewed as interpolating between the extremes of the spot Libor and terminal numeraires.

Since $B_{m}^{*}$ can evidently be realized by a self-financing trading strategy, $B_{m}^{*} / B_{N+1}$ is a positive martingale under the terminal measure $P^{N+1}$. Define a new measure $P_{m}^{*}$ by setting

$$
\left(\frac{d P_{m}^{*}}{d P^{N+1}}\right)_{t}=\frac{B_{m}^{*}(t) B_{N+1}(0)}{B_{N+1}(t) B_{m}^{*}(0)}
$$

Lemma 3 The dynamics of forward Libor under $P_{m}^{*}$ are as follows: for $n=$ $1, \ldots, m-1$,

$$
\frac{d L_{n}}{L_{n}}=-\sum_{i=n+1}^{m-1} \frac{\delta L_{i} \lambda_{i} \lambda_{n}^{\prime}}{1+\delta L_{i}} d t+\lambda_{n} d W_{t}
$$

and for $n=m, \ldots, N$

$$
\frac{d L_{n}}{L_{n}}= \begin{cases}\sum_{i=m}^{n} \frac{\delta L_{i} \lambda_{i} \lambda_{n}^{\prime}}{1+\delta L_{i}} d t+\lambda_{n} d W_{t} & \text { on }\left[0, T_{m}\right) \\ \sum_{i=\eta}^{n} \frac{\delta L_{i} \lambda_{i} \lambda_{n}^{\prime}}{1+\delta L_{i}} d t+\lambda_{n} d W_{t} & \text { on }\left[T_{m}, T_{N+1}\right]\end{cases}
$$

In particular, $L_{m-1}$ is a martingale under $P_{m}^{*}, m=2, \ldots, N+1$.

To construct a discretization, we introduce

$$
Z_{n} \equiv Z_{n}^{(m)}= \begin{cases}L_{n} \prod_{i=n+1}^{m-1}\left(1+\delta L_{i}\right), & n=1, \ldots, m-1, \\ L_{n} \prod_{i=m \vee \eta}^{n}\left(1+\delta L_{i}\right)^{-1}, & n=m, \ldots, N+1,\end{cases}
$$


with the convention that $L_{N+1} \equiv \lambda_{N+1} \equiv 0$. Clearly, $Z_{n}$ coincides with $X_{n}$ from Sect. 3.1 for $n<m$ and with $V_{n} / \delta$ from Sect.3.2 for $n \geq m$. (We could alternatively have constructed $Z_{n}$ for $n \geq m$ to parallel $D_{n}$; this would lead to an alternative discretization.)

Lemma 4 The $Z_{n}$ are martingales and satisfy $d Z_{n}=Z_{n} \sigma_{Z_{n}} d W$ with

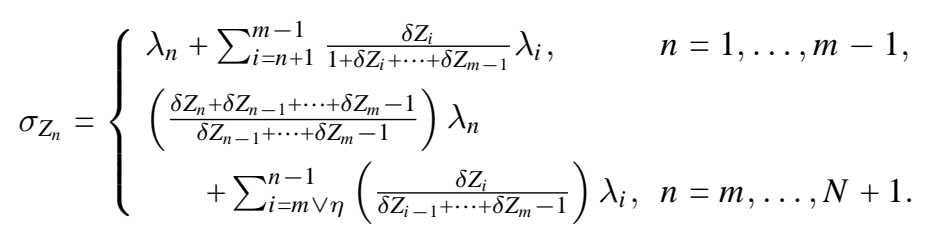

Also,

$$
L_{n}= \begin{cases}\frac{Z_{n}}{1+\delta Z_{n+1}+\cdots+\delta Z_{m-1}} & n=1, \ldots, m-1, \\ \frac{Z_{n}}{1-\delta Z_{n}-\cdots-\delta Z_{m}} & n \geq \eta \vee m\end{cases}
$$

The discretization scheme suggested by this result sets

$$
\hat{Z}_{n}((i+1) h)=\hat{Z}_{n}(i h) \exp \left(-\frac{1}{2} \sigma_{\hat{Z}_{n}}(i h) \sigma_{\hat{Z}_{n}}(i h)^{\prime}+\sigma_{\hat{Z}_{n}}(i h) \sqrt{h} \xi_{i+1}\right)
$$

(with $\sigma_{\hat{Z}_{n}}$ equal to $\sigma_{Z_{n}}$ but with $Z$ and $\lambda$ replaced by $\hat{Z}$ and $\hat{\lambda}$ ) and then applies (47) to recover discretized Libor rates $\hat{L}_{n}$ from $\hat{Z}_{n}$. We have chosen the representation in (47) to be consistent with (21) for $n \leq m-1$ and consistent with (40) for $n \geq m$, though we could consider other combinations of the schemes in Sections 3.1 and 3.2. The properties of this discretization are minor modifications of the ones in Theorems 1 and 2; we therefore record only the most notable feature in the following result:

Proposition 1 Fix $m \in\{2, \ldots, N+1\}$. Under the discretization above of $\left(L_{1}, \ldots, L_{N+1}\right)$ in the $P_{m}^{*}$ measure, $\hat{L}_{m-1}$ is a martingale,

$$
\delta B_{m}^{*}(0) E\left[\left(\hat{L}_{m-1}\left(T_{m-1}\right)-K\right)^{+}\right]=C\left(\bar{\lambda}_{m-1}\left(T_{m-1}\right), K, L_{m-1}(0), B_{m}^{*}(0), T_{m}\right) ;
$$

i.e., the $(m-1)$ th caplet is priced without discretization error.

Thus, the discretization of this section provides the flexibility to pinch the caplet bias to zero at any maturity, while simultaneously constructing forward Libor rates of all maturities and making the discretized deflated bond prices martingales.

\section{Discretization of forward swap rates}

We now turn to the discretization of the term structure of forward swap rates, starting from the continuous-time constructions of Jamshidian [14, 15]. Jamshidian [14] constructs all forward swap rates associated with a tenor structure, in particular allowing each forward swap rate to have a lognormal volatility so that 
swaptions are priced by Black's formula. He carries out the construction in both the terminal and spot Libor measures. We consider the discretization of each of these cases. Although the guiding principles in this setting are the same as in the forward Libor setting, the expressions involved tend to be more complicated. As a consequence, our results in this section are somewhat less explicit than in the previous sections.

\subsection{Swap rates in the terminal measure}

Our notation largely follows Jamshidian $[14,15]$. Let

$$
B_{n, N}(t)=\delta \sum_{i=n}^{N} B_{i+1}(t), \quad t \in\left[0, T_{n+1}\right]
$$

the $n$th forward swap rate, $n=1, \ldots, N$, is

$$
S_{n}(t)=\frac{B_{n}(t)-B_{N+1}(t)}{B_{n, N}(t)}, \quad t \in\left[0, T_{n}\right] .
$$

This is the fixed rate at time $t$ that equates the present values of streams of fixed-rate payments $\delta S_{n}(t)$ and floating-rate payments $\delta L_{i}\left(T_{i}\right)$ occurring at $T_{i+1}$, $i=n, \ldots, N$. We extend the definition of $S_{n}$ to all of $\left[0, T_{N+1}\right]$ by setting $S_{n}(t)=$ $S_{n}\left(T_{n}\right)$ for $t>T_{n}$. Forward Libor rates can be recovered from forward swap rates through the relation

$$
1+\delta L_{n}(t)=\frac{1+\delta S_{n} \sum_{i=n}^{N} \prod_{j=n+1}^{i}\left(1+\delta S_{j}\right)}{1+\delta S_{n+1} \sum_{i=n+1}^{N} \prod_{j=n+2}^{i}\left(1+\delta S_{j}\right)}
$$

(from Sect. 7 of [14]), and in fact (48) could be inverted to define forward swap rates in terms of forward Libor rates. Expressions for deflated bond prices in terms of forward swap rates follow from (48), using (6) and (9).

A lognormal (or market) model of forward swap rates specifies

$$
\frac{d S_{n}}{S_{n}}=\ldots d t+\theta_{n} d W
$$

for some bounded, deterministic, possibly time-varying row vector $\theta_{n}=\theta_{n}(t)$ having at most finitely many discontinuities. The drift depends on the choice of numeraire asset - i.e., the measure under which the process is constructed. The defining property of the terminal measure is the choice of $B_{N+1}$ as the numeraire asset. In this measure, Jamshidian [14] shows that the missing drift in (49) is given by $\alpha_{n}(t) \theta_{n}^{\prime}(t)$ with

$$
\alpha_{n}(t)=-\frac{\sum_{i=n}^{N} \sum_{k=n+1}^{i} \delta S_{k}(t) \theta_{k}(t) \prod_{j=n+1, j \neq k}^{i}\left(1+\delta S_{j}(t)\right)}{\sum_{i=n}^{N} \prod_{j=n+1}^{i}\left(1+\delta S_{j}(t)\right)} .
$$


He also shows that $S_{n}$ is a martingale under the forward swap measure $P^{n, N}$ defined by

$$
\left(\frac{d P^{n, N}}{d P^{N+1}}\right)_{t}=\frac{B_{n, N}(t) B_{N+1}(0)}{B_{N+1}(t) B_{n, N}(0)}
$$

this is the measure associated with the numeraire $B_{n, N}$.

A payer's swaption expiring at time $T \leq T_{n}$ grants the holder the right to enter into a swap with start date $T_{n}$ and end date $T_{N+1}$, making fixed payments $\delta K$ and receiving floating payments $\delta L_{i}\left(T_{i}\right)$ at $T_{i+1}, i=n, \ldots, N$. Its value at expiration can thus be expressed as $B_{n, N}(T)\left(S_{n}(T)-K\right)^{+}$. Jamshidian [14] shows that with a lognormal specification of the volatility

$$
\begin{aligned}
B_{N+1}(0) E^{N+1}\left[B_{n, N}(T)\left(S_{n}(T)-K\right)^{+} / B_{N+1}(T)\right] & =B_{n, N}(0) E^{n, N}\left[\left(S_{n}(T)-K\right)^{+}\right] \\
& =C\left(\bar{\theta}_{n}(T), K, S_{n}(0), B_{n, N}(0), T\right),
\end{aligned}
$$

with

$$
\bar{\theta}_{n}^{2}(t)=\frac{1}{t} \int_{0}^{t} \theta_{n}(u) \theta_{n}^{\prime}(u) d u ;
$$

i.e., swaptions are priced by the Black formula for swaptions. (See Musiela and Rutkowski [20] and Rebonato [22] for further discussion of this and related formulas for swaptions.)

It is evident from (48) that forward Libor rates cannot have lognormal volatilities if the forward swap rates do. Hence, one cannot use the discretizations of Sect. 3 to simulate a model consistent with (49). To discretize (49) in the terminal measure, we introduce the variables

$$
X_{n}=\frac{B_{n, N}}{\delta B_{N+1}}, \quad \text { and } \quad Y_{n-1}=X_{n-1}-X_{n}-1, \quad n=1, \ldots, N,
$$

with $X_{0}=1+\left(1+\delta S_{1}\right) X_{1}$. Notice that $Y_{n-1}=D_{n}-1=D_{n}-D_{N+1}$; this particular difference of deflated bond prices turns out to be particularly convenient and effective in discretizing swap rate models.

Lemma 5 Under the terminal measure, $X_{n}$ and $Y_{n}$ are martingales on $\left[0, T_{n+1}\right]$ and satisfy

$$
d X_{n}=\sum_{i=n+1}^{N}\left[\theta_{i}\left(X_{i-1}-X_{i}-1\right) \prod_{j=n+1}^{i-1} \frac{X_{j-1}-1}{X_{j}}\right] d W
$$

and

$$
\begin{aligned}
\frac{d Y_{n}}{Y_{n}}= & \left(\theta_{n+1}+\frac{1}{N-n+\sum_{k=n+1}^{N-1} Y_{k}}\right. \\
& \left.\times\left[\sum_{i=n+2}^{N} \theta_{i} Y_{i-1} \prod_{j=n+2}^{i-1}\left(\frac{Y_{j-1}}{N-j+1+\sum_{k=j}^{N-1} Y_{k}}+1\right)\right]\right) d W .
\end{aligned}
$$


Also,

$$
\delta S_{n}=\frac{X_{n-1}-X_{n}-1}{X_{n}}=\frac{Y_{n-1}}{N-n+1+\sum_{i=n}^{N-1} Y_{i}}
$$

The natural counterpart to the discretization methods developed in Sect. 3 applies the modified Euler scheme to $\log Y_{n}$ to construct discrete martingales $\hat{Y}_{n}(i h), i=0,1, \ldots$, and then applies (51) to recover discretized forward swap rates $\hat{S}_{n}$. One could use (48) to construct discretized forward Libor rates as well. We summarize properties of this method in the following result:

Theorem 3 (i) The discretization above makes the deflated bond prices positive martingales and is thus arbitrage-free. (ii) The $\hat{S}_{n}$ remain strictly positive, almost surely. (iii) Each $\hat{S}_{n}$ is a martingale with respect to the measure $d \hat{P}^{n, N}=\left(\delta B_{N+1}(0) \hat{X}_{n} B_{n, N}(0)\right) d P^{N+1}$. (iv) If $T$ is a grid point ih, then $B_{N+1}(0) \delta E\left[\left(\hat{S}_{N}(T)-K\right)^{+}\right]=C\left(\bar{\theta}_{N}, K, S_{N}(0), B_{n, N}(0), T\right)$; i.e., the last swaption is priced without discretization error. (v) The $\hat{S}_{n}$ converge in law to the $S_{n}$ as $h \rightarrow 0$.

\subsection{Swap rates in the spot Libor measure}

A consequence of Jamshidian's [14] analysis is that in the spot Libor measure the missing drift in (49) is $\left(\gamma_{\eta}-\alpha_{n}\right) \theta_{n}$ with $\alpha_{n}$ as in (50) and

$$
\gamma_{n}=\frac{\delta S_{n} \sum_{i=n}^{N}\left(\theta_{n}+\sum_{k=n+1}^{i} \delta \theta_{k} S_{k}\right) \prod_{j=n+1, j \neq k}^{i}\left(1+\delta S_{j}\right)}{1+\delta S_{n} \sum_{i=n}^{N} \prod_{j=n+1}^{i}\left(1+\delta S_{j}\right)} .
$$

As this might suggest, the relevant expressions in this setting become algebraically cumbersome, though they do not raise any fundamentally new issues. Consequently, we cover only the key steps.

For purposes of discretization we introduce the variables

$$
M_{n}=\frac{1+\delta S_{n} \sum_{i=n}^{N} \prod_{j=n+1}^{i}\left(1+\delta S_{j}\right)}{1+\delta S_{1} \sum_{i=1}^{N} \prod_{j=2}^{i}\left(1+\delta S_{j}\right)}, \quad n=1, \ldots, N+1,
$$

$R_{1}=M_{N+1}$, and $R_{n}=M_{n}-M_{N+1}, n=2, \ldots, N$. Under our convention that $S_{j}(t)=S_{j}\left(T_{j}\right)$ for $t>T_{j}$, these processes are well-defined throughout the interval $\left[0, T_{N+1}\right]$.

Lemma 6 Under the spot Libor measure, the $M_{n}$ and $R_{n}$ are positive martingales. Moreover,

$$
\delta S_{n}=\frac{M_{n}-M_{N+1}}{M_{n+1}+\cdots+M_{N+1}}=\frac{R_{n}}{R_{n+1}+\cdots+R_{N}+(N+1-n) R_{1}} .
$$

Using (49), (50), (52), and (53) we can find the diffusion coefficients $\sigma_{M_{n}}$ and $\sigma_{R_{n}}$ of $\log M_{n}$ and $\log R_{n}$ as functions of the $\theta_{j}$ and $S_{j}$ through straightforward but tedious differentiation. Using (54) we can then express $\sigma_{M_{n}}$ purely as functions of the $\theta_{j}$ and $M_{j}$ and we can express $\sigma_{R_{n}}$ purely as a function of the $\theta_{j}$ and 
$R_{j}$. In this way, we arrive at equations of the form $d M_{n} / M_{n}=\sigma_{M_{n}} d W$ and $d R_{n} / R_{n}=\sigma_{R_{n}} d W$ analogous to those derived in previous sections. The resulting expressions for $\sigma_{M_{n}}$ and $\sigma_{R_{n}}$ are lengthy and unenlightening so we omit them. Nevertheless, we may simulate an Euler scheme for $\log M_{n}$ or $\log R_{n}$ to produce discretized variables $\hat{M}_{n}$ or $\hat{R}_{n}$ and then use (54) to recover discretized swap rates $\hat{S}_{n}$.

Several features of these schemes are easily verified. Both ensure that deflated bond prices are positive martingales. For it follows from (9) and (48) that the discrete deflated bond prices are

$$
\begin{aligned}
\hat{D}_{n} & =B_{1}(0) \frac{1+\delta \hat{S}_{n} \sum_{i=n}^{N} \prod_{j=n+1}^{i}\left(1+\delta \hat{S}_{j}\right)}{1+\delta \hat{S}_{1} \sum_{i=1}^{N} \prod_{j=2}^{i}\left(1+\delta \hat{S}_{j}\right)} \\
& =B_{1}(0) \hat{M}_{n}=B_{1}(0)\left(\hat{R}_{n}+\hat{R}_{1}\right),
\end{aligned}
$$

and the $\hat{M}_{n}$ or $\hat{R}_{n}$ are positive martingales by construction. In addition, recovering the discretized swap rates from the $\hat{R}_{n}$ through (54) ensures that the swap rates stay positive.

As a final observation, we consider the discrete counterpart of the forward swap measure resulting from the discretization of $\log M_{n}$. In continuous time, the forward swap measure $P^{n, N}$ is related to the spot Libor measure through

$$
\left(\frac{d P^{n, N}}{d P^{*}}\right)_{t}=\frac{B_{n, N}(t) B^{*}(0)}{B^{*}(t) B_{n, N}(0)}=\left(\delta \sum_{i=n+1}^{N+1} D_{i}(t)\right) \frac{B^{*}(0)}{B_{n, N}(0)} .
$$

We therefore define

$$
d \hat{P}^{n, N}=\left(\delta \sum_{i=n+1}^{N+1} \hat{D}_{i}\right) \frac{B^{*}(0)}{B_{n, N}(0)} d P^{*} .
$$

Now

$$
\sum_{i=n+1}^{N+1} \hat{D}_{i}=B_{1}(0)\left(\hat{M}_{n+1}+\cdots+\hat{M}_{N+1}\right)=B_{1}(0)\left(\hat{R}_{n+1}+\cdots \hat{R}_{N}+(N+1-n) \hat{R}_{1}\right)
$$

so we see from (54) that under either discretization $\hat{S}_{n} \sum_{i=n+1}^{N+1} \hat{D}_{i}$ is a martingale under $P^{*}$ and thus $\hat{S}_{n}$ is a martingale under $\hat{P}^{n, N}$.

\section{Numerical comparison}

Thus far, our discussion of discretization has focused primarily on the extent to which the absence of arbitrage is preserved after discretization. To a lesser extent, we have considered the impact on the accuracy of caplet and swaption prices, the prices to which a model is commonly calibrated. The purpose of this section is to show, through numerical examples, that the discretizations proposed in previous sections do not entail a loss of accuracy in pricing caplets and swaptions. On the 
contrary, we find that these discretizations often introduce lower discretization bias in these prices than more obvious schemes. In addition, we investigate the effect of making a finite-sample adjustment that exploits the martingale property of deflated bond prices. In our experiments, this reduces the mean square error in caplet and swaption price, in some cases by vast amounts. The applicability of this method depends crucially on our approach to discretization.

The number of variations of methods, scenarios, and instruments one could investigate numerically is limitless. Our objective here is by no means to be exhaustive. In particular, among the interesting possibilities not explored here is the use of the many higher-order schemes in Kloeden and Platen [17] with either the variables introduced in Sections 3 and 4 or the forward Libor and swap rates themselves.

\subsection{Libor models}

The base scenario we use for most of our results sets $\delta=h=0.25$ and $N+1=40$, corresponding to a ten-year term structure of quarterly rates. The initial values are of the form $L_{n}(0)=\log (a+b n)$, with $a$ and $b$ chosen so that $L_{0}(0)=0.05$ and $L_{39}(0)=0.07$. The base-case volatilities are constant over the intervals $\left[T_{i}, T_{i+1}\right)$, with

$$
\lambda_{n}\left(T_{i}\right)=0.1500+0.0025(n-i), \quad i=0, \ldots, n-1, \quad n=1, \ldots, 39 .
$$

These values for the initial forward rates $L_{n}(0)$ and initial implied volatilities $\lambda_{n}(0)$ are broadly consistent with the U.S. dollar term structure in late 1997. The assumption that volatility depends only on time to maturity is made for simplicity. In addition to this base case, we will consider a few other scenarios. We compare the discretizations of Sect. 3 with Euler schemes for $L_{n}$ and $\log L_{n}$ (as in (11) and (12)). We mainly compare performance of the methods in pricing at-the-money caplets - the strike for the $n$th caplet is $K=L_{n}(0)$. In the base scenario, the prices for these caplets increase with $n$ from 3.74 to 23.77.

Figure 1 compares the estimated bias in caplet prices for four methods in the terminal measure: the $X_{n}$ discretization of (24), the $D_{n}$ discretization of (30), and Euler schemes for $L_{n}$ and $\log L_{n}$. The error bars have a halfwidth of one standard error and show that the apparent biases are highly significant and not attributable to simulation error. As expected, the $X_{n}$ and $\log L_{n}$ discretizations produce unbiased estimates of the final caplet. But for shorter maturities the $X_{n}$ method produces substantially smaller errors. The method in (30) does not appear competitive. Because it makes $1+\delta \hat{L}_{N}$ lognormal rather than $L_{N}$, it does not correctly price the final caplet. But in considering Fig. 1 and subsequent figures it is important to keep an additional consideration in mind: the martingale discretizations (including $X_{n}$ and $D_{n}$ in Fig. 1) price bonds without bias precisely because they make the deflated bond prices martingales. The other methods (including $L_{n}$ and $\log L_{n}$ in Fig. 1) are subject to discretization bias in pricing bonds as well as caplets. 


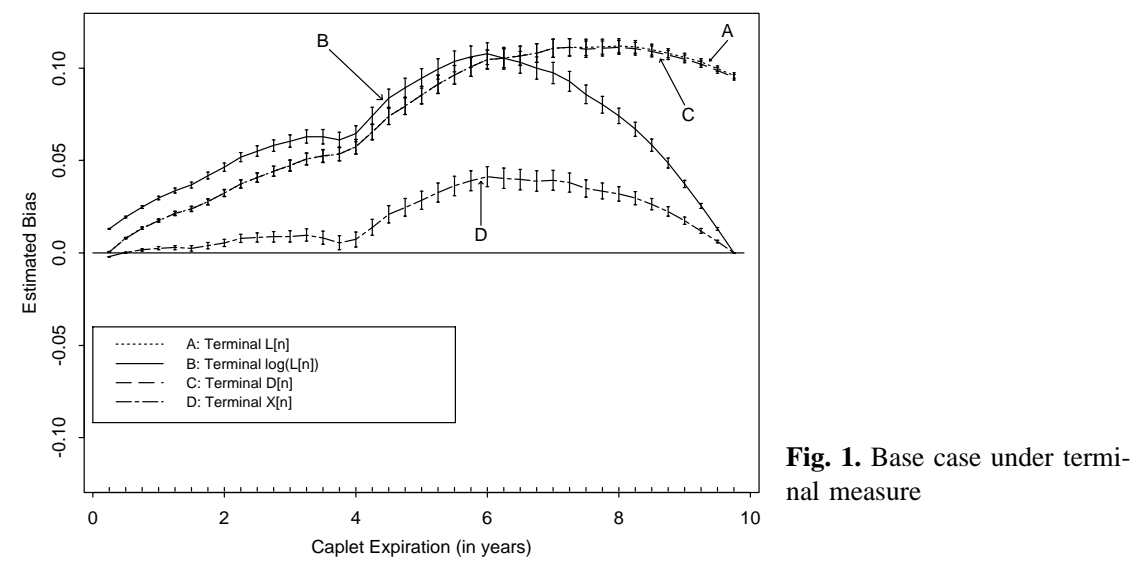

To obtain the tight error intervals in Fig. 1, we ran 10 million replications and used the control variates $C_{n}=\delta B_{n+1}(0)\left(\zeta_{n}-K\right)^{+}$where

$$
\zeta_{n}=L_{n}(0) \exp \left(-\frac{1}{2} h \sum_{i=0}^{n-1} \lambda_{n}^{2}(i h)+\sqrt{h} \sum_{i=0}^{n-1} \lambda_{n}(i h) Z_{i}\right),
$$

and the $Z_{i}$ are the standard normals used to drive the simulations. Each $C_{n}$ is an unbiased estimator of the $n$th caplet price. For any discretization $\hat{L}_{n}$, our estimate of the bias in the $n$th caplet is

$$
\delta B_{N+1}(0)\left(\hat{L}_{n}\left(T_{n}\right)-K\right)^{+} \hat{D}_{n+1}\left(T_{n+1}\right)-C_{n} .
$$

Because of the high correlation between the $\zeta_{n}$ and the discretized variables, subtracting $C_{n}$ rather than the Black price itself substantially reduces the variance of the estimated bias. We used this method in all our results.

Figure 2 compares five discretizations in the spot Libor measure. The $V_{n}$ method refers to (38) with the second line of (37) and (40); the modified $V_{n}$ method refers to (38) with the first line of (37) using (42) to determine the discrete deflated bond prices; the $D_{n}$ method refers to (39); and the other two are Euler schemes for $L_{n}$ and $\log L_{n}$. As in the terminal measure, the results based on $D_{n}$ and $L_{n}$ are very similar; and whereas these are biased high, discretization of $\log L_{n}$ produces results biased low. The $V_{n}$ method and modified $V_{n}$ methods give the best results across all maturities. Because the $V_{n}$ method does somewhat better than the modified $V_{n}$ method, we use it in subsequent comparisons.

Figure 3 illustrates the effect of using the hybrid numeraire of Sect. 3.3. The terminal measure corresponds to $m=N+1$ and the spot measure to $m=1$. These are compared with two intermediate values of $m$. As anticipated, the $(m-1)$ th caplet is priced without bias in each case for which $m>1$. (Notice the change of scale compared with the previous figures.) In this and most subsequent figures we omit the error bars for clarity; they are of the same magnitude as in Figures 1 and 2 . 


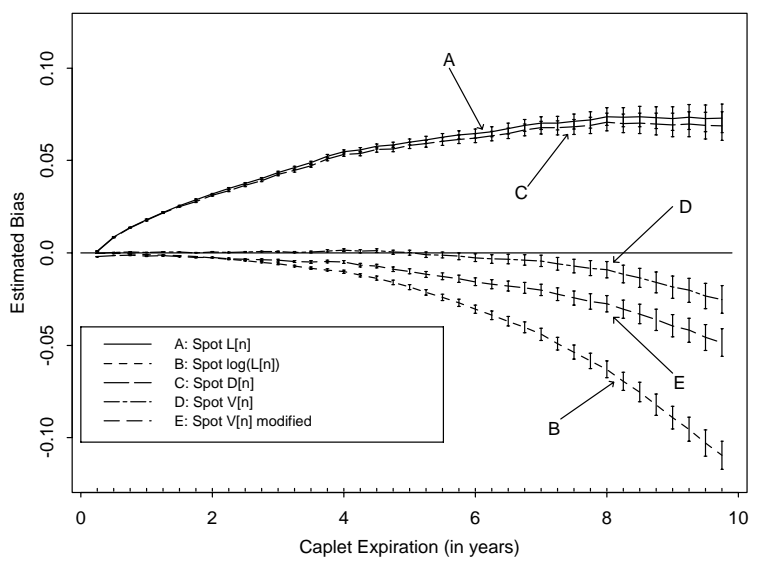

Fig. 2. Base case under spot measure

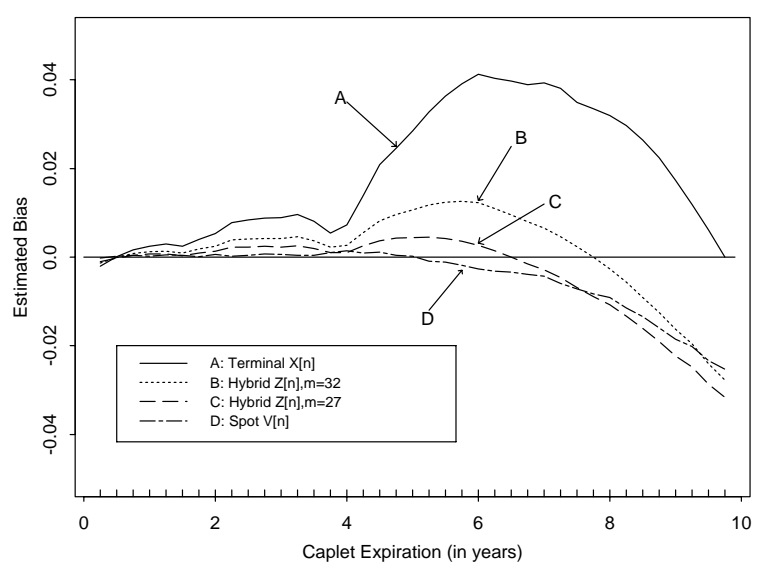

Fig. 3. Hybrid numeraire

Figures 4 and 5 compare various methods for caplets that are $5 \%$ in-themoney (strikes of $0.95 L_{n}(0)$ ) and out-of-the-money (strikes of $1.05 L_{n}(0)$ ), respectively. The results are generally consistent with what we observe at-the-money, except for some additional bias at very short maturities. Over most of the range, the $X_{n}$ and $V_{n}$ methods appear to be the best.

Figure 6 compares select methods in a variant of our base case in which the volatilities are $\lambda_{n}\left(T_{i}\right)=0.25-0.0025(n-i)$ and are thus decreasing rather than increasing with maturity. In Fig. 7 we again use increasing volatilities but now take $N=19, \delta=0.5, h=\delta / 2$, and $L_{n}(0)=\log (a+b n)$ with $a$ and $b$ chosen so that $L_{0}(0)=0.05$ and $L_{19}(0)=0.07$. Neither of these modifications affects the relative performance of the methods.

\subsection{Finite-sample adjustment}

As previously noted, discretizations that make the deflated bond prices martingales (and are initialized to the initial term structure) price bonds without 


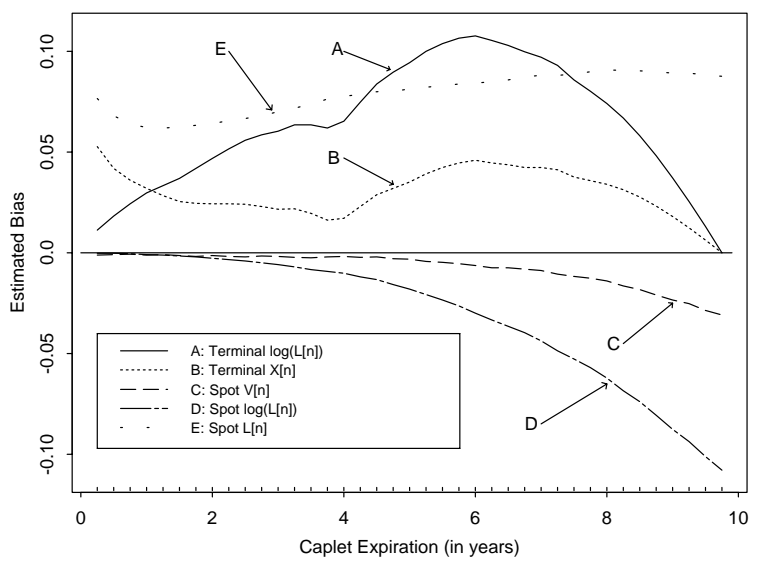

Fig. 4. In-the-money caplets

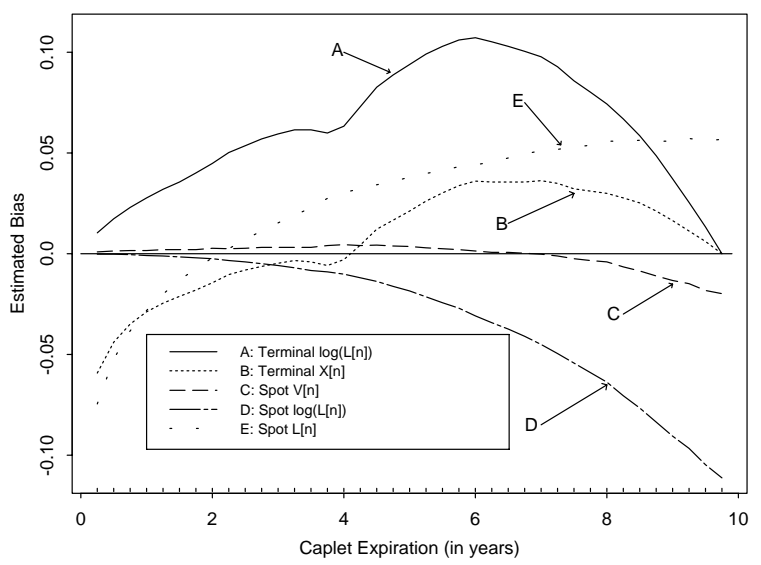

Fig. 5. Out-of-the-money caplets

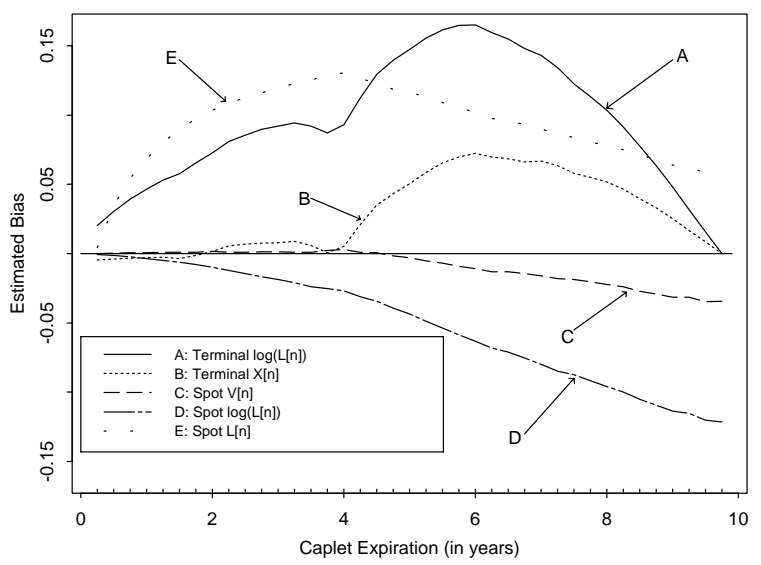

Fig. 6. Decreasing term structure of volatility 


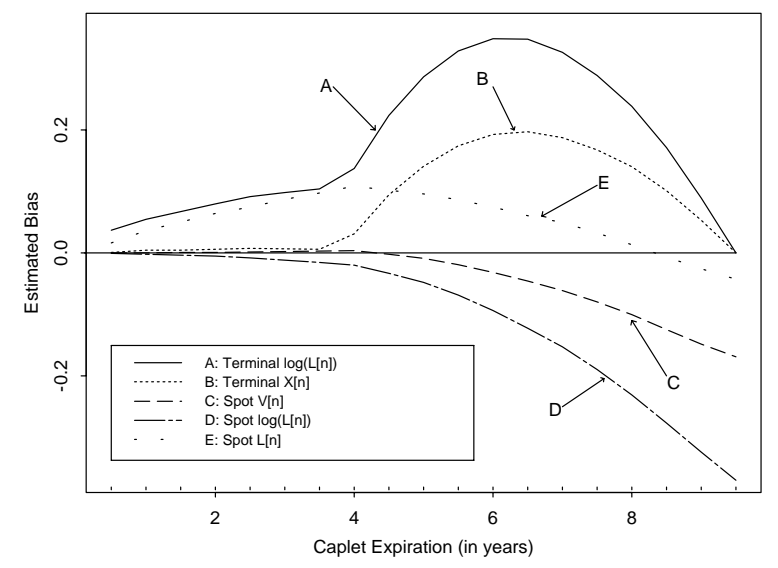

Fig. 7. Semi-annual rates

discretization bias. We now take this idea one step further and introduce a finitesample adjustment that makes the simulated bond prices match true bond prices over finitely many paths. We then examine the impact of this adjustment on caplet prices. A similar adjustment is proposed in a setting without discretization error by Duan and Simonato [6]; see also the discussion in Boyle et al. [4]. Similar adjustments appear to be in widespread use in practice.

We detail the method in the case of the discretization $\hat{X}_{n}$ in the terminal measure. Let $\hat{X}_{n}^{(k)}(i h), k=1, \ldots, K$, denote the values of $\hat{X}_{n}$ simulated at the $i$ th step of $K$ independent replications. Because each $\hat{X}_{n}$ is a martingale, $E\left[\hat{X}_{n}(i h)\right]=$ $\hat{X}_{n}(0)=X_{n}(0)$. To match this mean over the $K$ paths, we set

$$
\hat{X}_{n}^{(k)}(i h) \longleftarrow \hat{X}_{n}^{(k)}(i h) \frac{K X_{n}(0)}{\sum_{j=1}^{K} \hat{X}_{n}^{(j)}(i h)}, \quad k=1, \ldots, K
$$

and then recover the discretized Libor rates $\hat{L}_{n}(i h)$ from these adjusted variables. A similar adjustment is possible with any of our discretizations. It does not appear to be applicable to a standard discretization of $L_{n}$ or $\log L_{n}$, because neither $E\left[L_{n}(t)\right]$ nor $E\left[\log L_{n}(t)\right]$ is readily available, and the discretized deflated bond prices will not be martingales.

Although this method causes bonds to be priced without simulation error over finitely many paths - and may therefore reduce variance in pricing other instruments - it potentially introduces bias in dividing by the sample mean in (55). To balance bias and variance we examine the impact on mean square error of caplet prices.

Figure 8 plots mean square errors with and without the finite sample adjustment using the $X_{n}$ method in the terminal measure and the $V_{n}$ method in the spot measure. These estimates are based on 5000 batches each consisting of $K=1000$ replications. (The finite-sample adjustment makes the $K$ paths in a single batch dependent so batching is necessary for proper estimation of mean square errors.) The results show modest improvement from the adjustment. 


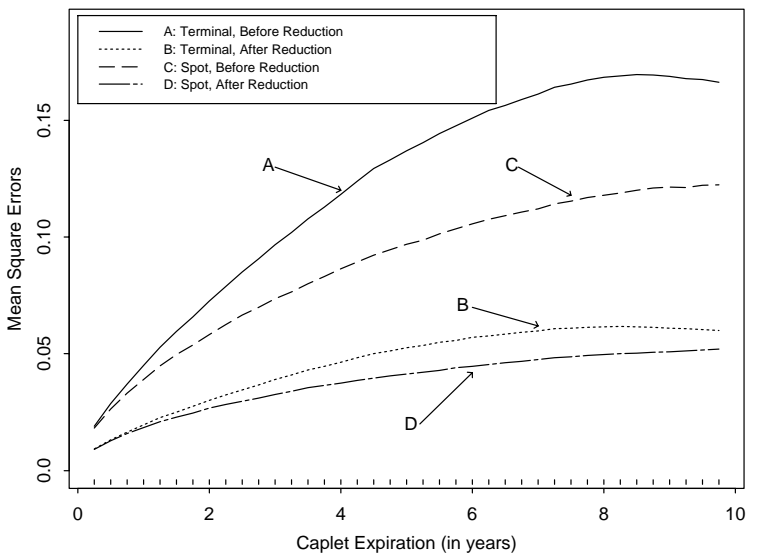

Fig. 8. Variance reduction in base case

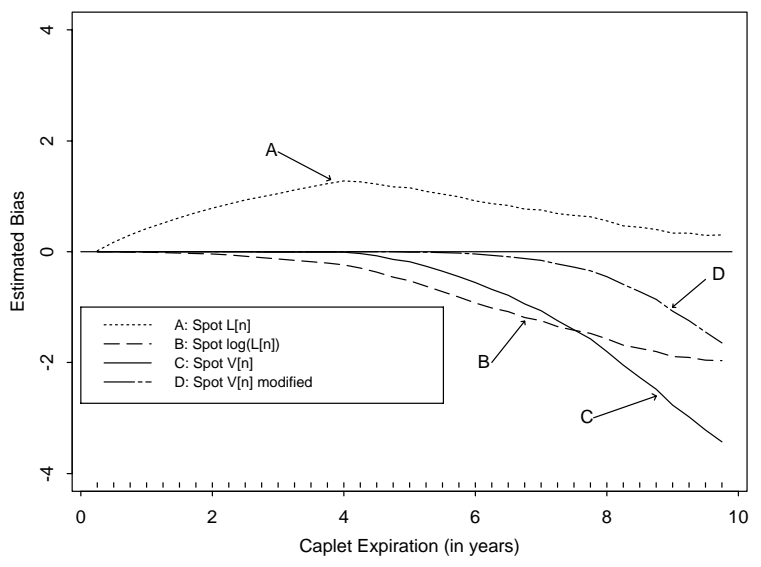

Fig. 9. JPY scenario - spot measure

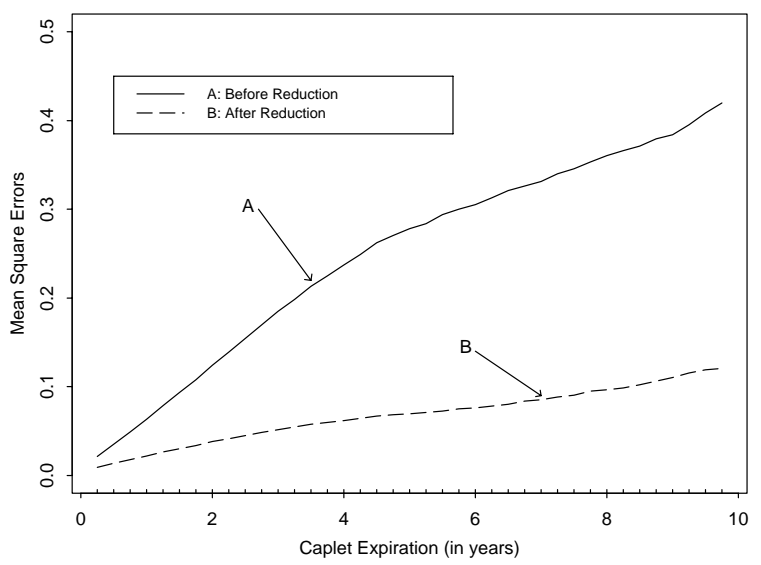

Fig. 10. Variance reduction in JPY spot measure 
We find much greater improvements in a different scenario. Here we take values broadly consistent with the Japanese ten-year term structure in late 1997. Specifically, $L_{n}(0)=0.01+0.00075 n, n=1, \ldots, 39$, and $\lambda_{n}(i)=0.70-0.01(n-$ $i$ ), so the rates are much lower and the volatilities much higher than in the U.S. market. The corresponding caplet prices increase with $n$ from 3.71 to 46.11 . These are rather extreme parameter values and no method appears to give satisfactory results in this setting. This is reflected in Fig. 9. Figure 10, based on the $V_{n}$ method in the spot measure, shows both that the errors in this setting can be quite large but also that the adjustment can produce substantial improvements. Figure 11 is even more dramatic. Over 1000 paths, the errors from simulating under the terminal measure are enormous, but they are brought under control by the finite-sample adjustment. The adjustment would not be available in a direct discretization of $L_{n}$ or $\log L_{n}$.

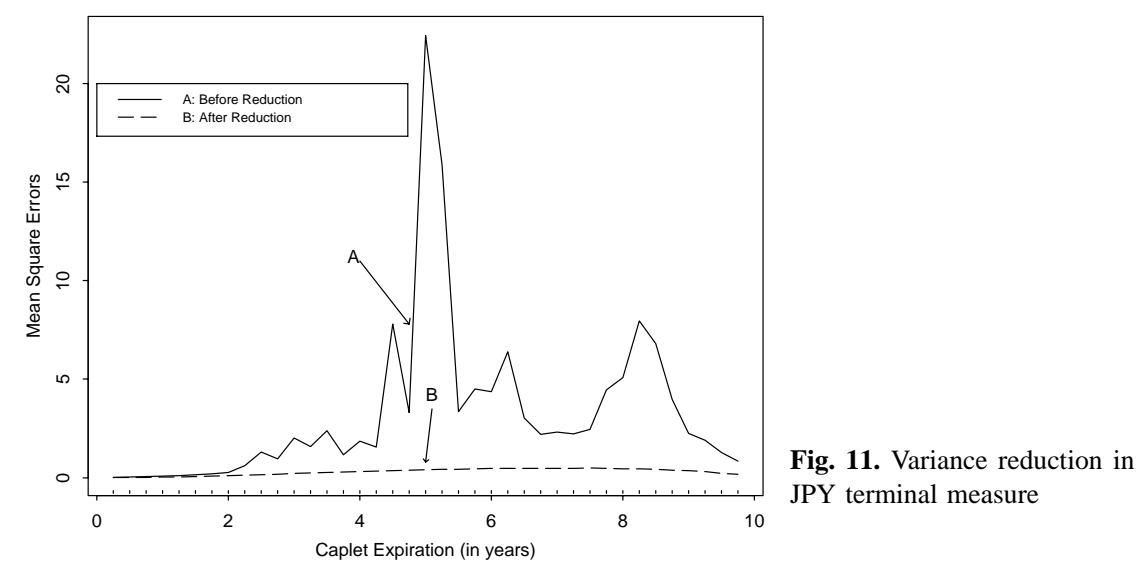

\subsection{Swap rate models}

Our base case for comparing discretizations of lognormal swap rate models uses the same initial term structure as in Sect.5.1 and specifies swap rate volatilities of

$$
\theta_{n}\left(T_{i}\right)=0.2475-(n-i) 0.0025, \quad i=1, \ldots, n-1, \quad n=1, \ldots, N .
$$

As should be clear from the formulas in Sect. 4, simulating any discretization of a lognormal swap rate model is considerably more demanding than simulating a lognormal model of forward Libor. Our investigations in this setting use fewer replications ( 1 million rather than 10 million) and have explored fewer alternatives.

Figure 12 summarizes the comparison of various methods. (These use a control variate similar to the one described in Sect. 5.1.) The worst methods are 


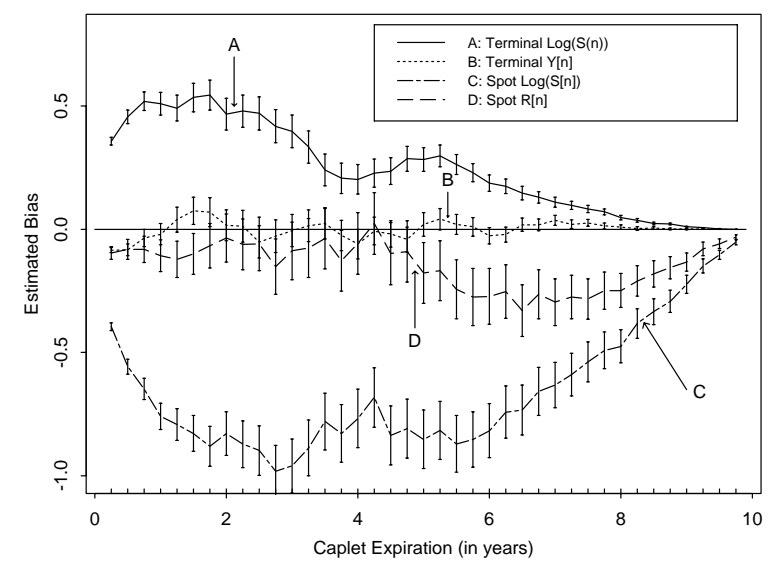

Fig. 12. Swaption pricing

the straightforward Euler discretizations of $S_{n}$ and $\log S_{n}$. The best method appears to be the $Y_{n}$ discretization under the terminal measure, but the spot Libor discretizations perform quite well also.

\section{Conclusions}

The main conclusion of this work is that simulating linear combinations of deflated bond prices can have advantages over direct simulation of forward Libor or swap rates. Linear combinations of deflated bond prices are martingales, and it is often easier to preserve the martingale property in a discretization scheme than to find the appropriate drift adjustment for a discretization of Libor or swap rates. By enforcing the martingale property in the discretization, we can keep the discretized model arbitrage-free. Our numerical results suggest that this advantage does not come at the expense of accuracy of the discretization; on the contrary, the schemes we have proposed generally give better results (and sometimes much better results) than more standard discretizations. They also lend themselves to a simple finite-sample adjustment that can further reduce errors.

We have by no means exhausted the possibilities opened up by this approach. One could investigate other linear combinations and other numeraire assets, linear combinations with time-varying coefficients, nonlinear transformations other than logarithms, variable step sizes, and any of the many higher-order methods developed in the discretization literature. Ruling out arbitrage does not, by itself, provide guidance in choosing among these many possibilities; we view these many variations and potential enhancements as complementing the approach proposed here.

\section{Appendix: Proofs}

Proof of Lemma 1. Equation (20) follows from (18) by induction, starting at $n=N$ (at which $X_{N}=L_{N}$ ) and proceeding down to $n=1$. Ito's lemma applied 
to (18) using (5) gives

$$
\frac{d X_{n}}{X_{n}}=\left(\lambda_{n}+\sum_{i=n+1}^{N} \frac{\delta L_{i} \lambda_{i}}{1+\delta L_{i}}\right) d W
$$

Substituting on the right using (20) gives (19). That $X_{n}$ is indeed a martingale follows from the boundedness of the expression in parentheses in (56).

Proof of Theorem 1. (i) That the $\hat{D}_{n}$ are positive martingales follows from (22) and the fact that the $\hat{X}_{i}$ are positive martingales by construction. (ii) Positivity of $\hat{L}_{n}$ follows from (21). (iii) Let $\hat{\mathscr{F}}_{i}$ be the $\sigma$-algebra generated by $\xi_{1}, \ldots, \xi_{i}$. For $i<j$,

$$
\begin{aligned}
\hat{E}^{n+1}\left[\hat{L}_{n}(j h) \mid \hat{\mathscr{F}}\right] & =E^{N+1}\left[\hat{L}_{n}(j h)\left(\frac{d \hat{P}^{n+1}}{d P^{N}}\right)_{j h} \mid \hat{\mathscr{F}}\right]\left(\frac{d \hat{P}^{n+1}}{d P^{N}}\right)_{i h}^{-1} \\
& =E^{N+1}\left[\hat{L}_{n}(j h) \hat{D}_{n+1}(j h) \mid \hat{\mathscr{F}} \hat{D}_{n+1}(i h)^{-1}\right. \\
& =\hat{L}_{n}(i h) \hat{D}_{n+1}(i h) \cdot \hat{D}_{n+1}(i h)^{-1}=\hat{L}_{n}(i h)
\end{aligned}
$$

using a standard change-of-measure identity for conditional expectations in the first equation, the definition of $\hat{P}^{n+1}$ in the second, and finally the fact that $\hat{L}_{n} \hat{D}_{n+1}=\hat{X}_{n}$ is a martingale under $P^{N+1}$.

(iv) Observe that $d X_{N}=X_{N} \lambda_{N} d W$ so

$$
X_{N}(t)=X_{N}(0) \exp \left(-\frac{1}{2} \bar{\lambda}_{N}^{2}(t) t+\int_{0}^{t} \lambda_{N}(u) d W_{u}\right) .
$$

Consequently, for $n=N$, (24) constructs the solution without discretization error. In particular, $\hat{X}_{N}(i h)$ has the distribution of $X_{N}(i h)$ at every grid point $i h$, and using the interpolation in (29) this property extends to intermediate times as well. But $L_{N} \equiv X_{N}$ and, under (21), $\hat{L}_{N} \equiv \hat{X}_{N}$, so $\hat{L}_{N}(t)$ has the distribution of $L_{N}(t)$ for all $t$, and then $E\left[\left(\hat{L}_{N}\left(T_{N}\right)-K\right)^{+}\right]=E\left[\left(L_{N}\left(T_{N}\right)-K\right)^{+}\right]$for all $K$.

(v) Define $\sigma_{n}: \mathbf{R}^{N} \times \mathbf{R}_{+} \rightarrow \mathbf{R}^{d}$ by

$$
\sigma_{n}(x, t)=x_{n}^{+}\left(\lambda_{n}(t)+\sum_{j=n+1}^{N} \frac{\delta x_{j}^{+} \lambda_{j}(t)}{1+\delta x_{j}^{+}+\cdots+\delta x_{N}^{+}}\right), \quad n=1, \ldots, N
$$

so that $d X_{n}(t)=\sigma_{n}(X(t), t) d W_{t}$. Set $\lambda_{\max }=\sup _{n, t}\left\|\lambda_{n}(t)\right\|$. Then each $\sigma_{n}$ is linearly bounded because

$$
\left\|\sigma_{n}(x, t)\right\| \leq\left|x_{n}\right| \sum_{j=n}^{N}\left\|\lambda_{j}(t)\right\| \leq N \lambda_{\max }\|x\|,
$$

and Lipschitz because

$$
\left\|\sigma_{n}(x, t)-\sigma_{n}(y, t)\right\| \leq 2 N \lambda_{\max }\|x-y\|,
$$


for all $x, y \in \mathbf{R}^{d}$. It now follows from Theorem II.2.13 of Gihman and Skorohod [9] that $\hat{X}$ converges in law to $X$ as $h \rightarrow 0$. Because the mapping from $\hat{X}$ to $\hat{L}$ is continuous and the same as the mapping from $X$ to $L$, it follows from Billingsley [2, Theorem 5.1, p.30] that $\hat{L}$ converges in law to $L$.

Proof of Lemma 2. From Jamshidian [14, 15], we know that the deflated bond prices $D_{n}$ are martingales. Moreover, $D_{n}=B_{1}(0) \prod_{1}^{n-1} Y_{i}$, so

$$
d D_{n}=B_{1}(0) \sum_{i=1}^{n-1}\left(Y_{i}-1\right) \lambda_{i} \prod_{j=1}^{n-1} Y_{j} d W,
$$

and

$$
\frac{d D_{n}}{D_{n}}=\sum_{i=1}^{n-1}\left(Y_{i}-1\right) \lambda_{i} d W,
$$

which becomes (32) upon substitution of $D_{i+1} / D_{i}$ for $Y_{i}$.

Since $V_{n}=B_{1}(0)\left[D_{n}-D_{n+1}\right]$, the $V_{n}$ inherit the martingale property from the $D_{n}$. Ito's lemma gives

$$
\frac{d V_{n}}{V_{n}}=\left(\lambda_{n} Y_{n}+\sum_{i=1}^{n-1}\left(Y_{i}-1\right) \lambda_{i}\right) d W .
$$

Solving for the $Y_{i}$ in terms of the $V_{j}$ we get

$$
Y_{i}=\frac{V_{n}-V_{n-1}-\cdots-V_{1}-1}{V_{n-1}-\cdots-V_{1}-1},
$$

and making this substitution yields (31). Straightforward induction arguments verify (33) and (34). To establish (35) observe that

$$
\sum_{n=1}^{N+1} V_{i}=\sum_{n=1}^{N}\left(\prod_{i=1}^{n-1} Y_{i}-\prod_{i=1}^{n} Y_{i}\right)+\prod_{i=1}^{N} Y_{i}=\prod_{i=1}^{0} Y_{i}=1,
$$

so (35) follows from (34).

Proof of Theorem 2. (i) That the $\tilde{D}_{n}$ are martingales is immediate from (39), that the $\hat{D}_{n}$ are martingales follows from (38) and (41), and that the $D_{n}^{\prime}$ are martingales follows from (38) and (44). (ii) Positivity of $\tilde{D}_{n}$ is also immediate from (39). In light of (41), positivity of $\hat{V}_{n}$ implies that the $\hat{D}_{n}$ decrease with $n$ in a scheme based on (40). Similarly, (44) and positivity of $\hat{V}_{n}$ imply that the $D_{n}^{\prime}$ are both positive and decreasing with $n$. (iii) Positivity of $L_{n}^{\prime}$ is a direct consequence of (42). (iv) From (34) we find that $\tilde{L}_{n} \tilde{D}_{n+1}=\left[\tilde{D}_{n}-\tilde{D}_{n+1}\right] / \delta$, so $\tilde{L}_{n} \tilde{D}_{n+1}$ is a martingale. Using the same argument as in Theorem 1(iii), this implies that $\tilde{L}_{n}$ is a martingale under $\tilde{P}_{n+1}$. Similarly, $L_{n}^{\prime} D_{n+1}^{\prime}$ is a martingale so $D_{n}^{\prime}$ is a martingale under $P_{n+1}^{\prime}$. (v) For $x \in \mathbf{R}^{N}$ and $t \in\left[0, T_{N+1}\right]$, let 


$$
\begin{aligned}
\sigma_{n}(x, t)= & x_{n}\left(\phi\left(\frac{x_{n}+x_{n-1}+\cdots+x_{1}-1}{x_{n-1}+\cdots+x_{1}-1}\right) \lambda_{n}(t)\right. \\
& \left.+\sum_{i=\eta(t)}^{n-1} \phi\left(\frac{x_{i}}{1-x_{i-1}-\cdots-x_{1}}\right) \lambda_{i}(t)\right),
\end{aligned}
$$

so that $d V_{n}(t)=\sigma_{n}(V(t), t) d W_{t}$. Just as in the proof of Theorem 1(iv), each $\sigma_{n}$ is linearly bounded and Lipschitz, so convergence in law of the $\hat{V}$ to $V$ follows from Theorem II.2.13 of Gihman and Skorohod [9]. Convergence in law of $\hat{L}$ and $L^{\prime}$ to $L$ then follows from the continuity of the mappings in (34) and (35). The same argument applies to $\tilde{D}$ and $\tilde{L}$.

Proof of Lemma 3. Let

$$
\xi_{t}=\left(\frac{d P_{m}^{*}}{d P^{N+1}}\right)_{t}=\frac{B_{m}^{*}(t) B_{N+1}(0)}{B_{N+1}(t) B_{m}^{*}(0)} .
$$

Then

$$
\xi_{t}= \begin{cases}B_{N+1}(0) \prod_{i=m}^{N}\left(1+\delta L_{i}(t)\right), & t \leq T_{m} \\ B_{N+1}(0) \prod_{i=m+1}^{\eta(t)} \frac{1}{B_{i}\left(T_{i-1}\right)} \prod_{i=\eta(t)}^{N}\left(1+\delta L_{i}(t)\right), & t>T_{m}\end{cases}
$$

Thus,

$$
\frac{d \xi_{t}}{\xi_{t}}=\left\{\begin{array}{l}
\sum_{i=m}^{N} \frac{\delta \lambda_{i}(t) L_{i}(t)}{1+\delta L_{i}(t)} d W_{t}, \quad t \leq T_{m} \\
\sum_{i=\eta(t)}^{N} \frac{\delta \lambda_{i}(t) L_{i}(t)}{1+\delta L_{i}(t)} d W_{t}, \quad t>T_{m}
\end{array}\right.
$$

It now follows from Girsanov's theorem that

$$
W_{t}^{o}=W_{t}-\int_{0}^{t} \sum_{i=m \vee \eta(s)}^{N} \frac{\delta \lambda_{i}^{\prime}(s) L_{i}(s)}{1+\delta L_{i}(s)} d s
$$

is a standard Brownian motion under $P_{m}^{*}$. In view of (5), this means that the dynamics of forward Libor under $P_{m}^{*}$ are given by

$$
\frac{d L_{n}(t)}{L_{n}(t)}= \begin{cases}\sum_{i=m \vee \eta(t)}^{n} \frac{\delta \lambda_{n}(t) \lambda_{i}(t)^{\prime} L_{i}(t)}{1+\delta L_{i}(t)} d t+\lambda_{n}(t) d W_{t}^{o}, & m \vee \eta(t) \leq n \\ -\sum_{i=n+1}^{m-1} \frac{\delta \lambda_{n}(t) \lambda_{i}(t)^{\prime} L_{i}(t)}{1+\delta L_{i}(t)} d t+\lambda_{n}(t) d W_{t}^{o}, & m \vee \eta(t) \geq n+1\end{cases}
$$

This is equivalent to the representation in the statement of the lemma.

Proof of Lemma 4. The calculations involved in verifying these expressions are very similar to those used in Lemmas 1 and 2 so we omit the details.

Proof of Proposition 1. The proofs of the two assertions in the proposition are essentially the same as the proofs given for parts (iii) and (iv) of Theorem 1.

Proof of Lemma 5. A simple induction argument verifies that

$$
X_{n}=1+\left(1+\delta S_{n+1}\right) X_{n+1}, \quad n=1, \ldots, N-1,
$$

from which follows 


$$
d X_{n}=\sum_{i=n+1}^{N}\left(\delta \theta_{i} S_{i} X_{i} \prod_{j=n+1}^{i-1}\left(1+\delta S_{j}\right)\right) d W
$$

Making the substitution $1+\delta S_{j}=\left(X_{j-1}-1\right) / X_{j}$ yields the expression in the lemma. For $Y_{n}$ we have

$$
\begin{aligned}
d Y_{n} & =d\left(X_{n}-X_{n+1}-1\right) \\
& =\left(\delta \theta_{n+1} S_{n+1} X_{n+1}+\sum_{i=n+2}^{N}\left[\delta \theta_{i} S_{i} X_{i} \delta S_{n+1} \prod_{j=n+2}^{i-1}\left(1+\delta S_{j}\right)\right]\right) d W .
\end{aligned}
$$

Noting that $\delta S_{n+1}=Y_{n} / X_{n+1}$, this becomes

$$
d Y_{n}=\left(\theta_{n+1} Y_{n}+\frac{Y_{n}}{X_{n+1}}\left[\sum_{i=n+2}^{N} \theta_{i} Y_{i-1} \prod_{j=n+2}^{i-1} \frac{X_{j-1}-1}{X_{j}}\right]\right) d W
$$

Finally making the substitution $X_{n}=\sum_{i=n}^{N-1} Y_{i}+N-n+1$ yields the expression in the lemma.

Proof of Theorem 3. (i) The deflated bond prices are given by $D_{n}=Y_{n-1}+1$, so the $\hat{D}_{n}$ are positive martingales because the $\hat{Y}_{n}$ are. (ii) Follows from (51) and positivity of the $\hat{Y}_{n}$. (iii) and (iv) are proved similarly to Theorem 1(iii)-(iv). (v) As in Theorems 1 and 2, the key step is verifying that the diffusion coefficient of $Y_{n}$ is linearly bounded and Lipschitz. In fact, from (58) we can see that the norm of the diffusion coefficient is bounded by a constant times the norm of $Y$. In particular, each of the factors $\left(X_{j-1}-1\right) / X_{j}$ is between 0 and 1 , because $Y_{j-1} \geq 0$; and each $Y_{i-1} / X_{n+1}$ is between 0 and 1 because $X_{n+1}=\sum_{i=n+1}^{N-1} Y_{i}+N-n+1$.

Acknowledgments. The authors thank the referees for their suggestions and their careful reading of the manuscript. This research is supported in part by National Science Foundation Grant DMI-9457189 and an IBM University Partnership Award.

\section{References}

1. Andersen, L.: Simulation and calibration of the HJM model. New York: General Re Financial Products 1995

2. Billingsley, P.: Convergence of probability measures. New York: Wiley 1968

3. Black, F.: The pricing of commodity contracts. J. Financial Econ. 3, 167-179 (1976)

4. Boyle, P., Broadie, M., Glasserman, P.: Simulation Methods for Security Pricing. J. Econ. Dynamics Control 21, 1267-1321 (1997)

5. Brace, A., Gatarek, D., and Musiela, M.: The market model of interest rate dynamics. Math. Finance 7, 127-155 (1997)

6. Duan, J.-C., Simonato, J.-G.: Empirical martingale simulation for asset prices. Manag. Sci. 9, 1218-1233 (1998) 
7. Duffie, D., Dynamic Asset Pricing Theory, 2nd edn. Princeton, NJ: Princeton University Press 1995

8. El Karoui, N., Geman, H., Rochet, J.C.: Changes of numeraire, changes of probability measure, and option pricing. J. Appl. Probab. 32, 443-458 (1995)

9. Gihman, I.I., Skorohod, A.V.: The Theory of Stochastic Processes, Vol. III. Berlin Heidelberg New York: Springer 1979

10. Goldys, B., Musiela, M., Sondermann, D.: Lognormality of rates and term structure models. Working paper, University of New South Wales 1994

11. Heath, D., Jarrow, R., Morton, A.: Bond pricing and the term structure of interest rates: a discrete time approximation. J. Financial Quantitat. Anal. 25, 419-440 (1990)

12. Heath, D., Jarrow, R., Morton, A.: Bond pricing and the term structure of interest rates: a new methodology for contingent claims valuation. Econometrica 60, 77-105 (1992)

13. Hull, J.C.: Options, Futures, and Other Derivatives, 3rd edn. Englewood Cliffs, NJ: Prentice-Hall 1997

14. Jamshidian, F.: LIBOR and swap market models and measures. Working paper, Sakura Global Capital, London, 1996

15. Jamshidian, F.: LIBOR and swap market models and measures. Finance Stochast. 1, 293-330 (1997)

16. Johnson, N.L.: Systems of frequency curves generated by methods of translation. Biometrika 36, 149-176 (1949)

17. Kloeden, P., Platen, E.: Numerical Solution of Stochastic Differential Equations. Berlin Heidelberg New York: Springer 1992

18. Milstein, G.N.: A method of second-order accuracy integration of stochastic differential equations. Theor. Probab. Appl. 19, 557-562 (1978)

19. Miltersen, K.R., Sandmann, K., Sondermann, D.: Closed-form solutions for term structure derivatives with lognormal interest rates. J. Finance 70, 409-430 (1997)

20. Musiela, M., Rutkowski, M.: Continuous-time term structure models: Forward measure approach. Finance Stochast. 1, 261-292 (1997)

21. Musiela, M., Rutkowski, M.: Martingale methods in financial modeling. Berlin Heidelberg New York: Springer 1997

22. Rebonato, R.: Interest-Rate Option Models. Chichester, UK: Wiley 1996

23. Sandmann, K., Sondermann, D.: A term structure model and the pricing of interest rate options. Rev. Futures Markets 12, 391-423 (1993)

24. Sandmann, K., Sondermann, D.: Log-normal interest rate models: stability and methodology. Discussion paper B-398, Department of Statistics, University of Bonn 1997

25. Sidenius, J.: LIBOR Market Models in Practice. Copenhagen: Skandinaviska Enskilda Banken 1997

26. Talay, D.: Efficient numerical schemes for the approximation of expectations of functionals of the solution of a S.D.E, and applications. In: Korezlioglu, H., Mazziotto, G., Szpirglas, J. (eds.) Filtering and Control of Random Processes. Lecture Notes in Control and Information Sciences, vol. 61. Berlin Heidelberg New York: Springer 1984 